\title{
A Data-Driven Model of an Emergency Department
}

\author{
Ward Whitt $^{1}$, Xiaopei Zhang ${ }^{2}$
}

\begin{abstract}
This paper develops an aggregate stochastic model of an emergency department (ED) based on a careful study of data on individual patient arrival times and length of stay in the ED of the Rambam Hospital in Israel, which was used in a large-scale exploratory data analysis by Armony et al. (2015). This data set is of special interest because it has been made publicly available, so that the experiments are reproducible. Our analysis confirms the previous conclusions about the time-varying arrival rate and its consequences, but we also find that the probability of admission to an internal ward from the ED and the patient length-of-stay distribution should be time varying as well. Our analysis culminates in a new time-varying infinite-server aggregate stochastic model of the ED, where both the length-of-stay distribution and the arrival rate are periodic over a week.
\end{abstract}

Keywords: emergency departments, nonstationary stochastic models, queueing models, nonhomogeneous Poisson process, time-varying length-of-stay distribution, two-time-scale arrival process model

\section{Introduction}

There is a long history of operations research studies aimed at improving the quality and efficiency of healthcare, as illustrated by the early study [1] and the recent surveys $[2,3,4,5]$. Nevertheless, as emphasized in [6], there remains a great need for further improvement. Much of this improvement

\footnotetext{
${ }^{1}$ Industrial Engineering and Operations Research, Columbia University, Email: ww2040@columbia.edu, Correspondence to: MailCode 4704, S. W. Mudd Building, 500 West 120th Street, New York NY 10027-6699, U.S.A.

${ }^{2}$ Industrial Engineering and Operations Research, Columbia University, Email: xz2363@columbia.edu
} 
is likely to come from vastly improved data collection, storage, retrieval and analysis.

\subsection{Publicly Available Data for Reproducible Studies}

The power of data analysis is illustrated by extensive exploratory data analysis of the patient flow in the large Rambam Hospital in Israel from a queueing science perspective conducted by Armony et al. [7]. In addition to their own analysis of the patient flow data at the level of individual patients, they arranged to make their data publicly available, thus facilitating reproducible studies aimed at generating general conclusions of widespread applicability. In this paper, we respond by analyzing a portion of the patient flow data provided by [7]. In particular, we focus on the emergency department (ED), just as in $\S 3$ of [7].

Among the many OR studies in healthcare, many have already focused on the emergency department, e.g., $[8,9,10,11,12]$. As those papers illustrate, the customary goal is to improve system design and operations. In contrast, in this paper, we focus solely on analyzing the patient flow data to determine what is a good aggregate stochastic model of the emergency department. This careful analysis is justified because emergency departments are complicated. The results here are intended to make it possible to more quickly build better stochastic models that can be used to improve healthcare design and operations.

The available ED patient flow data is powerful in that it includes arrival and departure times of individual patients, but it is also limited in that it does not contain a detailed account of all the steps and processes that take place during a patient's stay. Thus, given the available data, we are only able to construct a relatively rough aggregate stochastic model, but even that can be useful and is not easy. Our model has only three components: (i) an arrival process model, (ii) an admission probability model, and (iii) a LoS model. All three are complicated, because we find that all three should be regarded as time-varying. Given those components, our aggregate stochastic model for system occupancy is a $G_{t} / G I_{t} / \infty$ time-varying infinite-server queue, which is much more tractable than the notation suggests. $\left(G_{t}\right.$ denotes a general (non-renewal, non-Markov) time-varying arrival process, while $G I_{t}$ denotes mutually independent service times, independent of the arrival process, but with a time-dependent distribution.) The $M_{t} / M_{t} / \infty$ infinite-server model was proposed for healthcare in 1976 by Collings and Stoneman [13]. 
Because of the limited data, the possible direct applications of the full model for operational improvement are limited. However, it can be used to perform "what if" studies, e.g., to estimate the performance impact of the arrival rate increasing by $3 \%$ per year over the next 5 years. As reviewed in [14], infinite-server models describe the time-varying load together with the drivers of that load. Of course, the load can be measured directly by the census, i.e., the number of patients in the ED as a function of time, but we expose how that is related to the main drivers, in particular, the patient arrival process and the length of stay (LoS) of the patients, both of which are time-varying, and should be regarded as stochastic. We think that the new model is most useful to provide new useful models of the principal model components, especially the patient arrival process. Almost any stochastic model used to model a healthcare system has a component that is a model of the patient arrival process.

\subsection{Time-Dependence}

As others have discovered before, e.g., see $\S 6.3$ of [15], the authors in $\S 3$ of [7] observe significant time dependence in the arrival rate and average occupancy levels of the ED. We confirm those observations here, but we go further by pointing out significant time dependence in (i) the probability of admission into an internal ward from the ED, (ii) the length-of-stay (LoS) distribution of arriving patients and (iii) the departure rate. Time dependence in LoS was also a major theme in the recent study of a Singapore hospital in [16]. We discuss the relation between time dependence and the state dependence emphasized by $[7,17]$ in $\S 4.5$.

Consistent with $\S 3$ of [7], we find that the ED arrival rate should be time varying, but we emphasize that the proper view is over a week as opposed to the common daily view. In particular, we think that the arrival rate can be regarded as periodic over a week. As in [7] and [11] before, we find that there is moderate overdispersion compared to a non-homogeneous Poisson process (NHPP). We conclude that it might be reasonable to use an NHPP arrival process model, but in fact we suggest instead a two-time-scale arrival process model. We suggest first modelling the daily totals as a discrete-time Gaussian process and then letting the arrivals during the day, given the daily total, be distributed as an NHPP. The conditional NHPP means that the arrival times (not interarrival times!) of the daily total number of arrivals are treated as i.i.d. random variables on the entire day with a probability density function (pdf) proportional to the arrival rate function for that day, as discussed in 
$[18,19]$. This arrival process model is a variant of the model proposed by [18]. We find that the model is supported by the statistical tests in [19, 20]; see $\S 3.4$.

The two-time-scale model is convenient because it supports focusing on arrivals over successive days and arrivals within days separately. Modeling the daily totals separately is convenient for applying time-series methods that can test and capture trends and stochastic dependence among successive days, but we did not detect strong evidence of it (beyond the significant dayof-week effect). There is precedent for two-time-scale healthcare models in $[16,21]$, but these are very different. The first [21] focuses on the hospital plus the ED, observing that the Internal Wards (IW's) operate on the slower time scale of days, whereas the ED operates on the faster time scale of hours. The paper [21] proposes and analyzes a Markov chain (MC) model of that system, using a discrete-time MC for the days and a continuous-time MC for the transitions within days. On the other hand, in $\S 3.2$ of [16] the authors propose a two-time-scale model of the LoS. The general thrust of [16] is consistent with our time-varying LoS distribution, which we discuss next.

We present strong evidence that the LoS distribution needs to be regarded as time-varying, and find that it suffices to make it time-varying over hours. Figure 8 here shows that the average occupancy level and departure rate are not predicted properly by a using the overall LoS distribution. In particular, there is a significant surge of departures just before midnight on each day as can be from Figures 8 (left) and 17. Moreover, these midnight departures occur for arrivals across a wide range of times, as can be seen from Table 5 . We can make the connection by applying the theory of infinite-server queues as in [22] or, equivalently, the time-varying Little's law [23, 24]. We show how the time-varying LoS can be efficiently and effectively analyzed by exploiting a discrete-time model in the time scale of hours. The time dependence in the LoS may prove useful in studying the scheduled operations in the ED and the internal wards.

\subsection{Organization}

In $\S 2$ we briefly describe the Rambam hospital and our data source, referring to [7] for more details. We analyze and model the ED arrival process in $\S 3$; we also discuss the probability of admission into an internal ward there. In $\S 4$, we analyze and model the LoS. In $\S 5$, we examine the departure process, showing that it can be useful to view the departure process in reverse time. In $\S 6$ we compare our model to simulation. Finally, we draw 
conclusions in $\S 7$. Supplementary material is provided in an online appendix [25].

\section{The Rambam Emergency Department and the Data}

As in [7], we study the Rambam hospital, a large 1000-bed hospital with 45 medical units in Haifa, Israel. In particular, as in $\S 3$ of [7], we focus on the emergency internal medicine unit (EIMU), which is the largest unit in a comprehensive emergency department (ED). That focus is justified because the different units of the ED are physically separate and share few resources. About $60 \%$ of all new patients enter the hospital through the ED and the majority of those enter through the EIMU, which we henceforth simply call the ED. After being examined and treated within the ED, patients are either admitted to one of the internal wards (IW's) or released, as depicted in Figure 2 of [7]. About $40 \%$ of arrivals to the ED are admitted.

As directed in Appendix 2 of [7], we obtained the data from the SEELab data-based research laboratory at the Technion. The available hospital data was collected from January 2004 to October 2007. We only focus on the 25-week period from December 2004 to May 2005. In particular, we use the 5 th, 6th, 13th and 18th columns of the visit table in the database, which are the entry group, first department, entry time and ED duration. In the raw data, the time records are rounded to the nearest second.

A total of 58,332 patients visited the comprehensive ED, with 24,317 going to the EIMU (3955, 4360, 3530, 4324, 3965 and 4183 for each month). Table 1 provides the total number of arrivals to the ED and length-of-stay (LoS) statistics for each of the sample populations used in successive analyses. The LoS refers to the LoS within the ED up until the time that a decision is made to admit the patient to an IW or not. Thus, the LoS does not include the delay until transfer is completed after the admission decision, commonly called ED boarding.

From both the database and [7], we know that the ED patients can be divided into two groups according to the admission decision; we pay attention to whether or not patients are admitted. Even though the admission decision cannot be known in advance, we find that the proportion of admitted patients in successive hours is time-dependent and thus can be exploited in modelling and analysis.

There are several variables in the database that can be used to help classify the patients. In this paper we use the "exit_group", which we find 
to be consistent with the "exit_unit", "exit_department" and "num_dep" in the visits table. "exit_group $=1$ " means the patient was released from the Emergency Department and was not admitted to any hospital department; "exit_group=2" means the patient was released from a hospital department, which means that he was admitted to at least one department from the ED. So when we focus on patients who entered ED first, this will tell us whether a patient was admitted to an IW or not. Among the 23, 409 patients that visited the ED (the EIMU) in the 25-week period, 9, 669 (about 40\%) were admitted and 13, 740 were not.

\begin{tabular}{|c|c|c|c|c|c|c|c|}
\hline No. & Data description & Sample Size & mean & stdv & 1st qu. & median & 3rd qu. \\
\hline 1 & Larger ED & $58,332(182$ days $)$ & 3.08 & 5.36 & 1.06 & 2.16 & 3.87 \\
\hline 2 & ED (EIMU) & $24,317(182$ days) & 4.10 & 3.49 & 1.90 & 3.31 & 5.26 \\
\hline 3 & Truncation of 2 & $23,409(175$ days $)$ & 4.10 & 3.49 & 1.90 & 3.31 & 5.26 \\
\hline 4 & Truncation of 2 & $23,421(175$ days) & 4.09 & 3.41 & 1.90 & 3.31 & 5.26 \\
\hline 5 & Admitted from 3 & $9,669(175$ days $)$ & 4.01 & 3.26 & 1.68 & 3.45 & 5.53 \\
\hline 6 & Non-admitted from 3 & $13,740(175$ days) & 4.17 & 3.65 & 2.01 & 3.24 & 5.05 \\
\hline 7 & Truncation of 2 & $23,407(175$ days $)$ & 4.10 & 3.50 & 1.90 & 3.31 & 5.27 \\
\hline 8 & Admitted from 7 & $9,668(175$ days $)$ & 4.01 & 3.27 & 1.68 & 3.45 & 5.53 \\
\hline 9 & Non-admitted from 7 & $13739(175$ days $)$ & 4.17 & 3.65 & 2.01 & 3.24 & 5.06 \\
\hline
\end{tabular}

Table 1: Sample size (number of arrivals) and LoS statistics (in hours) in different views of the ED data.

Below is a detailed description of the datasets in Table 1. Throughout the paper, we will refer to Table 1 to specify the population.

- Dataset 2: a subset of dataset 1 containing those patients who went to the EIMU within the comprehensive ED during the 25 week period; we refer to the EIMU simply as the ED.

- Dataset 3: a subset of dataset 2 containing those patients that arrived within the 25 week period. (We use dataset 2 for occupancy statistics.)

- Dataset 4: a subset of the dataset 2 containing those patients who both entered and departed the system between Dec. 5, 2004, and May 28, 2005, and have LOS less then 48 hours.

- Dataset 5: a subset of dataset 3 containing those patients who were admitted after visiting the ED ("exit_group=1").

- Dataset 6: a subset of dataset 3 containing those patients who were not admitted after visiting the ED ("exit_group=2"). 
- Dataset 7: a subset of dataset 2 containing those patients whose departure times are in the 25-week period (from Dec. 5, 2004, to May 28, 2005).

- Dataset 8: a subset of dataset 7 containing those patients who were admitted after visiting the ED ("exit_group=1").

- Dataset 9: a subset of dataset 7 containing those patients who were not admitted after visiting the ED ("exit_group=2").

\section{The ED Arrival Process}

In this section, we study the arrival process of patients at the ED (by which we always mean the EIMU). In $\S 3.1$ we look at the daily totals; we briefly discuss dependence among the daily totals in $\S 3.2$. In $\S 3.3$ we estimate the hourly arrival rates over a week. We evaluate the stochastic variability in the arrival process in $\S 3.4$, which leads to proposing the two-time-scale model involving a conditional nonhomogeneous Poisson process (NHPP). In §3.4.1 we estimate the index of dispersion for counts; in $\S 3.4 .2$ we report results of statistical tests of the conditional NHPP property, drawing on [19, 20]. In $\S 3.5$ we examine the arrival processes of two separate groups of patients: those that were ultimately admitted to one of the IW's and those that were not. Finally, in $§ 3.6$, we summarize the two-time-scale model for the arrival process that we propose, based on that statistical analysis.

\subsection{Daily Totals}

Table 2 shows the number of patients that arrived at the ED on each day from Dec. 5, 2004, to May 28, 2005 (25 weeks). The $25 \times 7=175$ daily totals vary from 77 to 191, and have mean 133.8 and median 135 .

Some of the fluctuation may be explained by Jewish holidays. In Israel, Dec. 8, 2004, to Dec. 12, 2004, in week 1 and 2 was Hanukkah, while Apr. 24, 2005, to Apr. 30, 2005, in week 21 was Passover. We see that the daily totals are somewhat low during weeks 1 and 2, but not very different in week 21. We also notice that another low period occurred between Feb. 4, 2005, and Feb. 11, 2005, in weeks 9 and 10, for which we have no explanation. It is possible that this was due to military hostilities, but we could not verify that. We do not omit these periods from our data because they represent unanticipated random events that do occur. 


\begin{tabular}{|c||c|c|c|c|c|c|c||c|c|}
\hline Week & Sun. & Mon. & Tue. & Wed. & Thu. & Fri. & Sat. & Total & Mean \\
\hline \hline 1 & 150 & 147 & 132 & 107 & 123 & 100 & 99 & 858 & 122.57 \\
\hline 2 & 143 & 147 & 127 & 138 & 121 & 101 & 103 & 880 & 125.71 \\
\hline 3 & 162 & 155 & 147 & 136 & 144 & 94 & 98 & 936 & 133.71 \\
\hline 4 & 186 & 155 & 135 & 136 & 119 & 100 & 131 & 962 & 137.43 \\
\hline 5 & 164 & 171 & 149 & 146 & 142 & 110 & 110 & 992 & 141.71 \\
\hline 6 & 175 & 144 & 157 & 136 & 156 & 115 & 105 & 988 & 141.14 \\
\hline 7 & 181 & 157 & 140 & 109 & 145 & 114 & 107 & 953 & 136.14 \\
\hline 8 & 176 & 145 & 139 & 150 & 126 & 127 & 102 & 965 & 137.86 \\
\hline 9 & 171 & 160 & 125 & 137 & 137 & 77 & 84 & 891 & 127.29 \\
\hline 10 & 134 & 127 & 119 & 115 & 95 & 88 & 82 & 760 & 108.57 \\
\hline 11 & 165 & 117 & 121 & 133 & 154 & 123 & 132 & 945 & 135.00 \\
\hline 12 & 163 & 142 & 135 & 142 & 129 & 115 & 100 & 926 & 132.29 \\
\hline 13 & 173 & 166 & 168 & 136 & 138 & 108 & 108 & 997 & 142.43 \\
\hline 14 & 169 & 155 & 155 & 137 & 143 & 127 & 106 & 992 & 141.71 \\
\hline 15 & 180 & 152 & 132 & 148 & 162 & 111 & 105 & 990 & 141.43 \\
\hline 16 & 159 & 164 & 191 & 128 & 126 & 95 & 111 & 974 & 139.14 \\
\hline 17 & 163 & 135 & 146 & 128 & 138 & 111 & 145 & 966 & 138.00 \\
\hline 18 & 160 & 123 & 168 & 136 & 133 & 119 & 102 & 941 & 134.43 \\
\hline 19 & 132 & 147 & 152 & 138 & 133 & 116 & 101 & 919 & 131.29 \\
\hline 20 & 162 & 150 & 140 & 126 & 113 & 113 & 96 & 900 & 128.57 \\
\hline 21 & 143 & 165 & 153 & 130 & 130 & 111 & 117 & 949 & 135.57 \\
\hline 22 & 151 & 147 & 132 & 114 & 114 & 114 & 96 & 868 & 124.00 \\
\hline 23 & 159 & 135 & 151 & 119 & 107 & 122 & 100 & 893 & 127.57 \\
\hline 24 & 164 & 163 & 153 & 147 & 156 & 111 & 91 & 985 & 140.71 \\
\hline 25 & 165 & 141 & 159 & 138 & 147 & 125 & 104 & 979 & 139.86 \\
\hline \hline Total & 4050 & 3710 & 3626 & 3310 & 3331 & 2747 & 2635 & 23409 & \\
\hline Mean & 162.00 & 148.40 & 145.04 & 132.40 & 133.24 & 109.88 & 105.40 & 936.36 & \\
\hline Var. & 191.58 & 187.08 & 275.71 & 139.33 & 270.44 & 152.78 & 196.75 & 3110.99 & \\
\hline & & & & & & & & & \\
\hline
\end{tabular}

Table 2: Number of arrivals at the ED on each day from Dec. 5, 2004, to May 28, 2005, (25 weeks, Dataset 2).

Within a week, Sunday has the largest number of arrivals, which is to be expected because it is the beginning of the work week in Israel. Then the average daily totals decrease over the week. Friday and Saturday have much fewer arrivals, which may be expected because that is the weekend. We also computed the variance of daily totals for each day of week. By looking at the dispersion (ratio of the variance to the mean), we see that there is a moderate level of overdispersion for the daily totals compared to a Poisson process (where the dispersion is 1).

Figure 1 is a plot of the weekly totals. It confirms our observation above about the low values in week 10 . Table 2 shows that the mean weekly number of arrivals is 936. Hence, if the arrival process were an NHPP, then the variance should be same as the mean and the standard deviation of the weekly total would be about $\sqrt{936} \approx 31$. Figure 1 is roughly consistent with that Poisson property, except for week 10, which is about 5 standard 


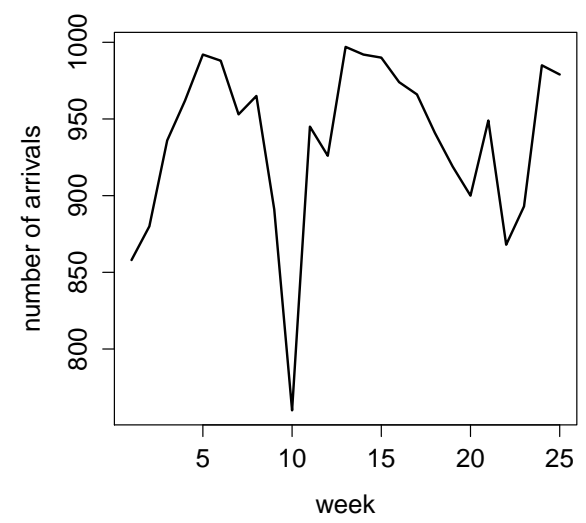

Figure 1: Weekly arrival totals over the 25-week study period. (Use dataset 3.)

deviations below the mean.

We investigated models for the daily totals. We first considered a twofactor statistical regression model with Gaussian residuals for the daily total numbers of arrivals; see $\S \S 2.7,3.7$ and 6.5 of [26] for background. The daily total is represented as

$$
T(w, d) \equiv A+B w+C d+G\left(0, \sigma^{2}\right),
$$

where $\equiv$ denotes equality by definition, $w$ represents the week and $d$ is the day-of-week (DoW), while $G\left(0, \sigma^{2}\right)$ is a mean-0 Gaussian random variable with variance $\sigma^{2}$ (to be estimated) and $A, B$ and $C$ are constants. The week and the DoW are the two factors, so actually we have $w_{i}$ 's as indicators for each week, $d_{j}$ 's as indicators for each day-of-week and $B_{i}$ 's, $C_{j}$ 's accordingly. Because there is redundancy in model (1) since $\sum_{i} w_{i}=1$ and $\sum_{j=1}^{7} d_{j}=1$, we set $\sum B_{i} \equiv 0$ and $\sum C_{j} \equiv 0$, so that $A$ gives the average daily total number of arrivals for all days.

Table 3 is the usual Analysis of Variance (ANOVA) table for the regression. From the $P$-values in the last column of Table 3 , we see that both factors are statistically significant at the $1 \%$ level. From the residuals, the estimated variance is $\hat{\sigma}^{2}=161.5=12.71^{2}$. Under this model, the variance-to-mean ratio is $161.5 / 133.8=1.21$. The Gaussian two-factor model is supported by observing that the residuals are consistent with the Gaussian distribution, as can be seen from the histogram of the residuals and the QQ-plot of the studentized residuals in the appendix [25]. 


\begin{tabular}{|r|r|r|r|r|r|}
\hline Factor & Sum of square & df & Mean sum of square & F statistics & P-value \\
\hline Week & 10,666 & 24 & 444.4 & 2.75 & $1.1 \times 10^{-4}$ \\
\hline DoW & 62,893 & 6 & $10,482.2$ & 64.89 & $<10^{-12}$ \\
\hline Residuals & 23,262 & 144 & 161.5 & & \\
\hline
\end{tabular}

Table 3: ANOVA table for the two-factor model (1). (Use dataset 3.)

However, for applications, we would actually prefer the single-factor model with only the DoW as the single factor, because the DoW effect is known, whereas the week effect is not, but the results above show the consequence if we can assume that it can be known or, more generally, if better estimates of the daily totals can be generated from forecasting. Hence, instead of (1), we propose the single-factor model

$$
T(d) \equiv A+C d+G\left(0, \sigma^{2}\right),
$$

where again $d$ represents the DoW factor and $G\left(0, \sigma^{2}\right)$ is the Gaussian random variable, while $A$ and $C$ are constants. Again, we set $\sum C_{j}=0$ to avoid redundancy.

Table 4 shows the estimated coefficients for model (2), while Figure 2 shows the histogram and QQ-plot for the residuals. The coefficients $C_{j}$ quantify the decreasing trend of the daily total arrivals within a week. Figure 2 shows that the normality of the residuals remains good. The ANOVA table can be computed from Table 3. The estimated variance and dispersion (variance-to-mean ratio) are

$$
\hat{\sigma}^{2}=\frac{10666+23262}{24+144}=202.0 \quad \text { and } \quad D \equiv \frac{\hat{\sigma}^{2}}{\hat{m}}=\frac{202.0}{133.8}=1.51 \text {, }
$$

where $\hat{m}$ is the estimated mean, which again represents a moderate level of overdispersion relative to an NHPP.

\subsection{Dependence Among Daily Totals and Residuals}

We also examined the dependence among the residuals in the singlefactor model. We first directly estimated the autocorrelation function and found the first seven coefficients were all positive. We then fit and compared autoregressive $\mathrm{AR}(\mathrm{p})$ models, and found that the fitting was not very good, but positive coefficients again indicate some positive dependence among the

residuals. Nevertheless, when we performed four different statistical tests of the residuals, we found that none could reject the independence hypothesis. 


\begin{tabular}{|c|r|c|}
\hline Coeffitients & Estimate & SE \\
\hline A & 133.766 & 2.842 \\
\hline C.Sun & 28.234 & 4.019 \\
\hline C.Mon & 14.634 & 4.019 \\
\hline C.Tue & 11.274 & 4.019 \\
\hline C.Wed & -1.366 & 4.019 \\
\hline C.Thu & -0.526 & 4.019 \\
\hline C.Fri & -23.886 & 4.019 \\
\hline C.Sat & -28.366 & 4.019 \\
\hline
\end{tabular}

Table 4: Estimated regression coefficients for the single-factor model in (2). (Use dataset 3.)
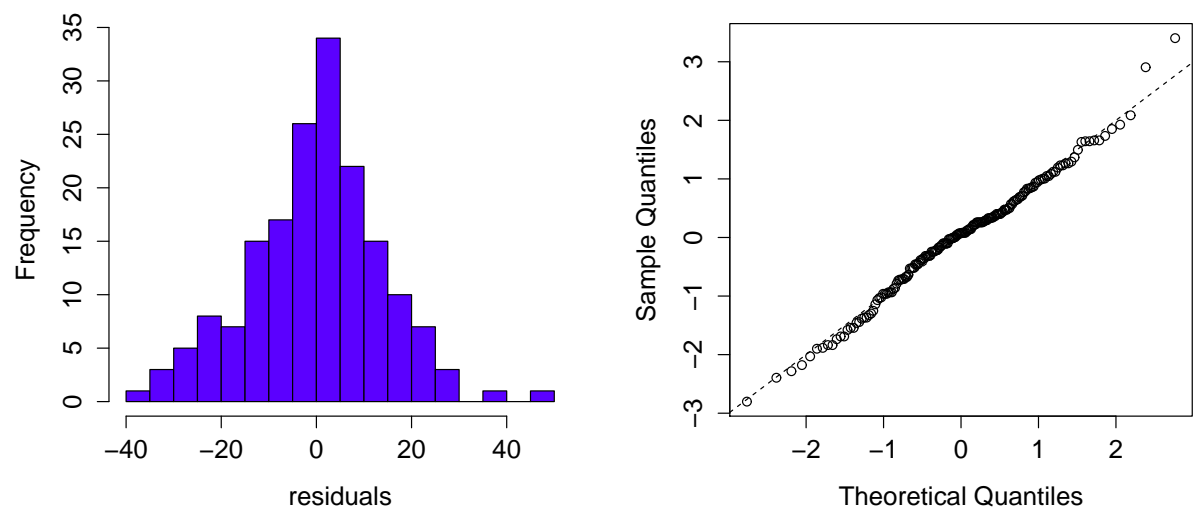

Figure 2: Supprting detail for model (2): histogram of the residuals (left) and QQ plot of studentized residuals (right). (Use dataset 3.)

Finally, we also fit $\operatorname{ARMA}(\mathrm{p}, \mathrm{q})$ models for the actual daily totals for various $p$ and $q$, with $p=7$ being a natural choice because of the observed DoW effect. Overall, we did not find a better model to suggest. Thus the details are left to the appendix [25].

\subsection{Arrival Pattern Within Days}

We now estimate the time-varying arrival rate by computing hourly averages and using a piecewise-linear plot. Unlike most service systems, we find that it is important to take a week view as opposed to a day view. Thus, we 
combine all the 25 weeks and estimate the hourly arrival rate over a week, as is shown in Figure 3. The vertical dashed lines are at midnight between successive days. Figure 3 shows that the arrival rate is lowest in the early morning, about 6am, and increases rapidly to a peak just before noon, after which it declines irregulary, with a steep decline around midnight. As expected, the arrival rate is lower at night than during the day. We can also see that the arrival rate is lower on weekends and has a somewhat different pattern.

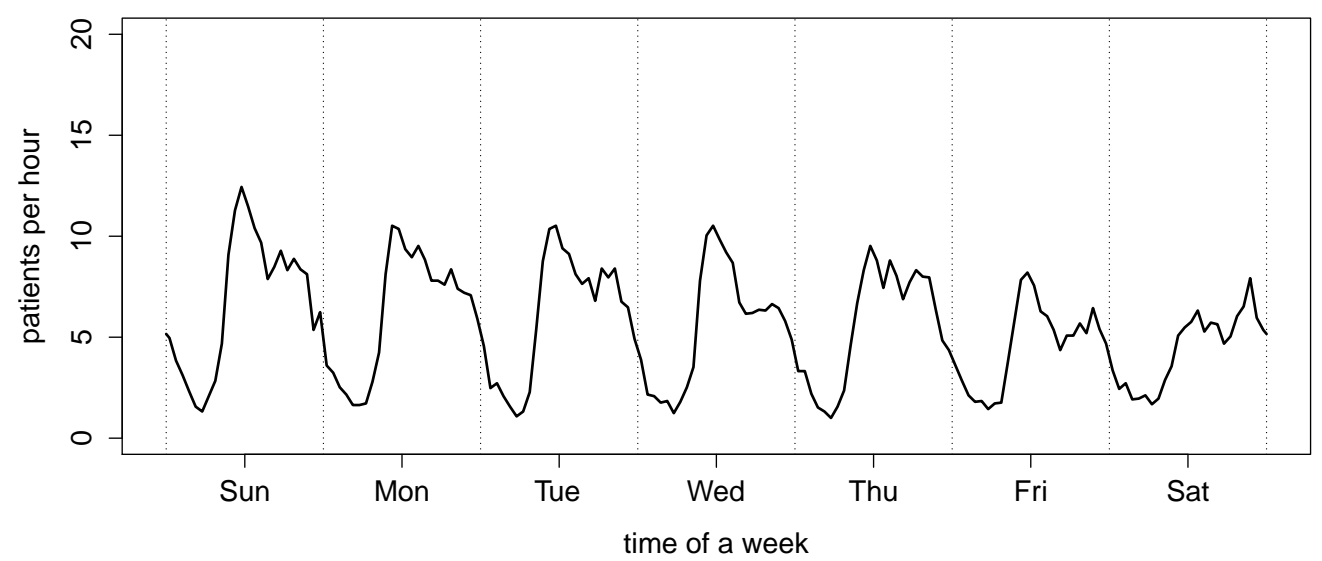

Figure 3: Estimated arrival rate at the ED over a week. (Use dataset 3.)

Since we have demonstrated a strong DoW effect on the daily totals, it is natural to examine the daily pattern without the DoW effect. To do so, we can normalize the arrival rate by the daily totals; i.e., we divide the arrival rate in Figure 3 by the average daily total arrivals of each day of week. Figure 4 (left) shows the arrival rate after normalizing, while Figure 4 (right) shows the corresponding estimated cumulative arrival rate function. Figure 4 shows that the normalized arrival rates still look different for different days, but we see more regular behavior with the cumulative view. Figure 4 suggests that it should not be unreasonable to approximate the arrival rate by a lower constant rate from midnight to 9am and a higher constant rate from 9am to midnight. This relatively simple arrival rate model is appealing, but we found that it did not perform as well in simulation comparisons. 
Figure 4: Estimated normalized arrival rate function over the week (left) and the corresponding estimate cumulative arrival rate function (right). These are both compared to a piecewise-constant approximation with two pieces divided at 9 am and midnight.
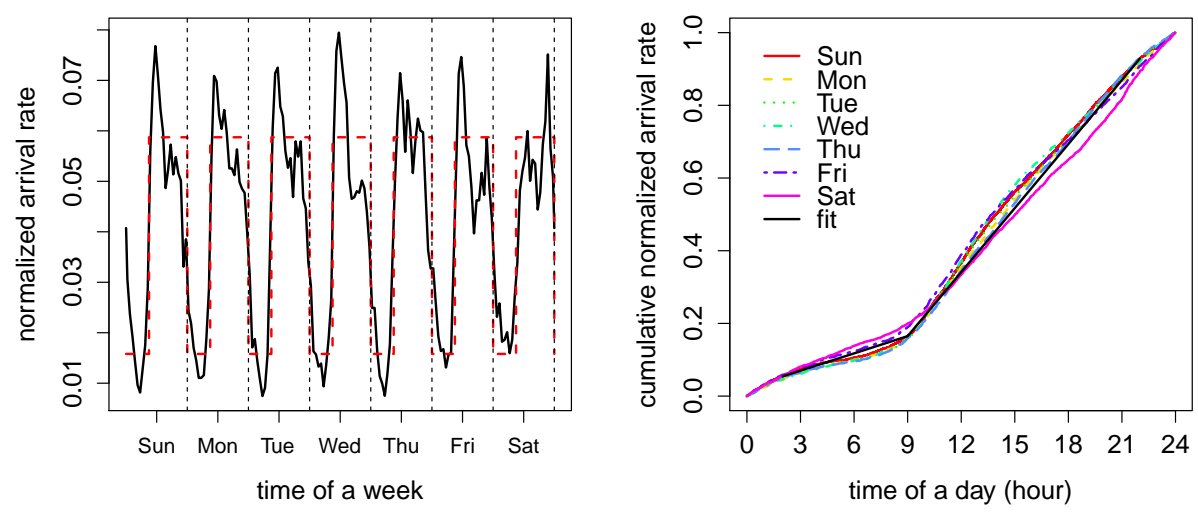

\subsection{Stochastic Variability in the Time-Varying Arrival Process}

It is commonly accepted that the arrival process to an ED can be modelled by a nonhomogeneous Poisson process (NHPP), because the arrivals typically come from the independent medical incidents of many different people, each of whom uses the ED infrequently. Mathematical support is provided by the Poisson superposition theorem; e.g., $§ 11.2$ of [27], but that should be verified, as in $[19,20]$.

Indeed, we have already seen strong stochastic variation in the daily totals that suggests overdispersion relative to a Poisson process. To illustrate unsuspected bunching of arrival that can occur, anecdotally from New York, ED employees report surges of arrivals at public transportation arrival times at the hospital.

Accordingly, we investigated the stochastic variability in the arrival process by (i) estimating the index of dispersion for counts, as in [28, 29], and by performing statistical tests of the NHPP property as in [19, 20]. We briefly summarize the results of our investigations and refer to the appendix for more details.

\subsubsection{The Index of Dispersion for Counts}

The index of dispersion for counts (IDC) is the ratio of the variance to the mean of the arrival counting process, as a function of time. Let $A(t)$ be 
the number of arrivals in interval $[0, t]$, so that $\{A(t),: t \geq 0\}$ is the arrival counting process. Let $\Lambda(t) \equiv \mathbb{E}[A(t)]$ and $V(t) \equiv \operatorname{Var}(A(t))$ be the mean and variance functions. Then the IDC is $I(t) \equiv V(t) / \Lambda(t), t \geq 0$.

It is instructive to consider three different views: (i) the week view, (ii) the day view and (iii) the DoW view. In the week view we take $T=7 * 24=168$ hours, and estimate $\Lambda(t)$ and $V(t)$ hourly by taking the 25 weeks as samples, then compute the ratio to estimate $I(t)$. In the day view we take $T=24$ hours, and take the $25 * 7=175$ days as samples. In DoW view we take $T=24$ hours, and take each specific day of week in the 25 weeks, so that the sample size is 25 for each day of week. Notice that it is natural to regard successive Tuesdays as i.i.d., but not successive days, so that the DoW view is likely to have less dependence.

Figure 5 shows estimates of the IDC in all three views. In both the week and day views IDC is steadily increasing, which reveals dependence over multiple days. In contrast, in the DoW view the IDC is much more flat, at a level that is not much greater than 1 for Poisson. The DoW view in Figure 5 shows that the average IDC is about 1.5, which coincides with the regression result for the daily total arrivals in $\S 3.1$. Figure 5 provides strong evidence that the overall arrival process is not too well modelled as an NHPP, but is quite well modelled as a conditional NHPP, where the arrival process conditional on the daily total is regarded as an NHPP. As explained in $§ 3.2$ of [30], that means that, after we condition on the daily total, those arrival times can be regarded as i.i.d. random variables over the day, each having a pdf proportional to the time-varying arrival-rate function.

Our analysis is consistent with the conclusions in [11] and in $\S 3.2$ of [7], but with very different method showing it. In [7], by exploratory data analysis, the authors found that the ED hourly arrival rates is time-varying. [11], which is also cited by [7], observed overdispersion for the arrival process by looking at empirical coefficients of variation in 4 time resolutions (hourly, 3-hour, 8-hour and daily). Here we go further by showing the time variability structure through the IDC.

\subsubsection{Statistical Tests of the NHPP Property}

To statistically test the deviations from the conditional NHPP assumption, we used the statistical tests in $[19,20]$, in particular, the conditional uniform Kolmogorov-Smirnov test (CU KS test) and the Lewis KS test. The test results are shown in the appendix. The results indicate that most intervals passed these KS tests, indicating that it is reasonable to regard the 

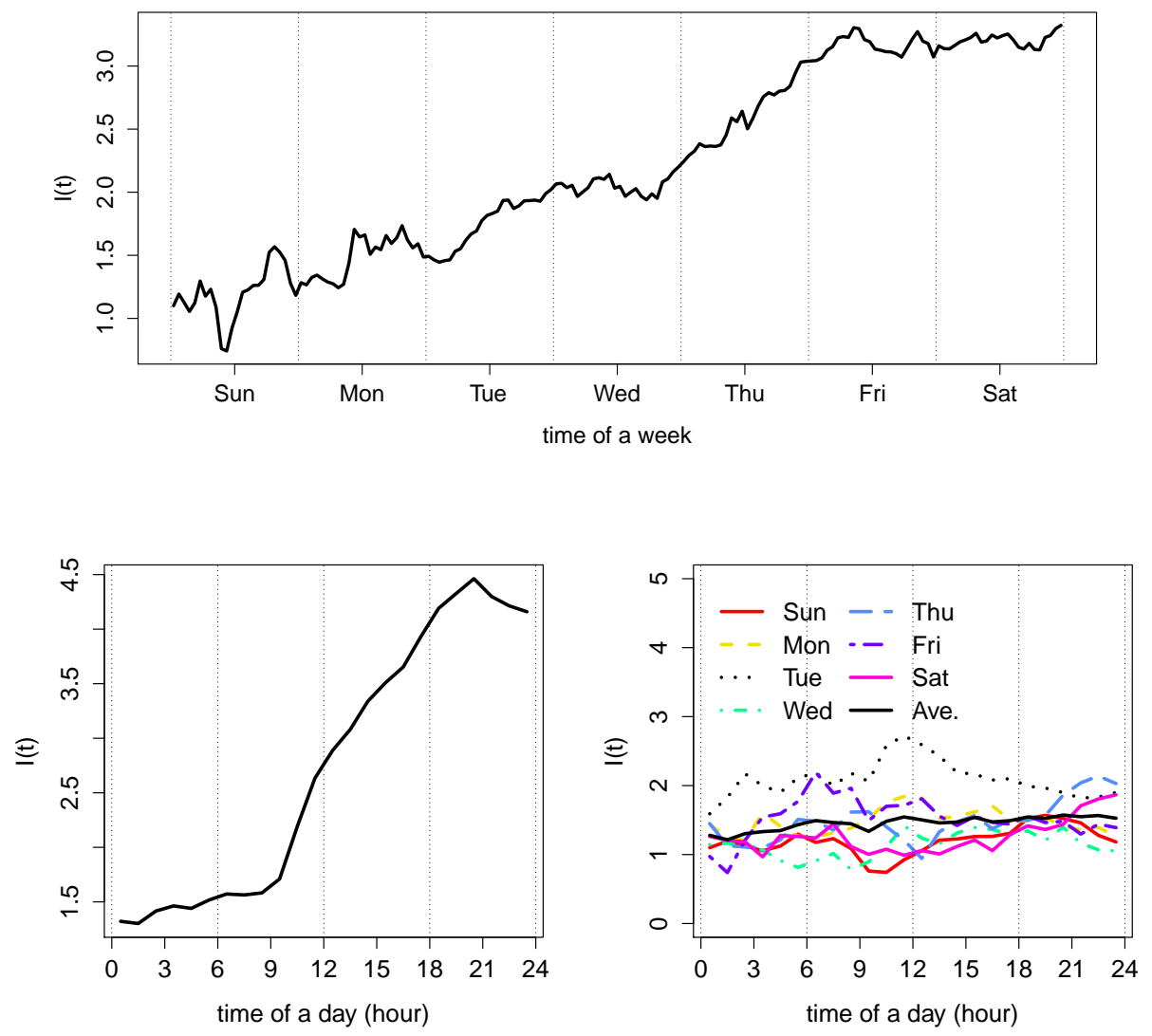

Figure 5: The estimated IDC in a week view (top), day view (bottom left) and DoW view (bottom right). (Use dataset 3.)

arrival processes as NHPP within each day. As emphasized in [19], that does not imply that the arrival-rate function should be regarded as deterministic. Instead, it supports the conditional NHPP property, because these statistical tests cannot distinguish between the conditional NHPP and the direct NHPP when the separate days are analyzed separately, as in the DoW view in the previous subsection.

In summary, we propose a two-time-scale model that has random daily totals and, conditional on those totals, assumes that the arrival process within each day is an NHPP. The conditioning feature means that, conditional on the daily totals, that number of arrivals is modelled as i.i.d. random vari- 
ables over the entire day, each having a pdf proportional to the arrival rate function. We use $M_{t}^{T}$ to denote this two-time-scale conditonal NHPP arrival process, where $T$ denotes conditioning on the daily totals. A variant of this $M_{t}^{T}$ arrival process model was proposed for appointment-generated arrival processes in [18]. For appointment-generated arrival processes, the arrival process tended to be under-dispersed compared to a Poisson process.

\subsection{Arrival Processes of the Two Groups: Admitted and Non-Admitted}

In $\S 2$ we mentioned that the patients in ED can be divided into two groups according to the admission decision (to the internal ward from the ED). The non-admitted patients are released after being treated in the ED while the admitted ones are transferred to the IW's in the main hospital. A priori, we judge that these two arrival processes can be regarded as an independent thinning from the whole arrival process. For managing ED's, we wanted to investigate if this thinning might be time-dependent. Figure 6 shows the estimated arrival rates of the admitted and non-admitted patients for a week.

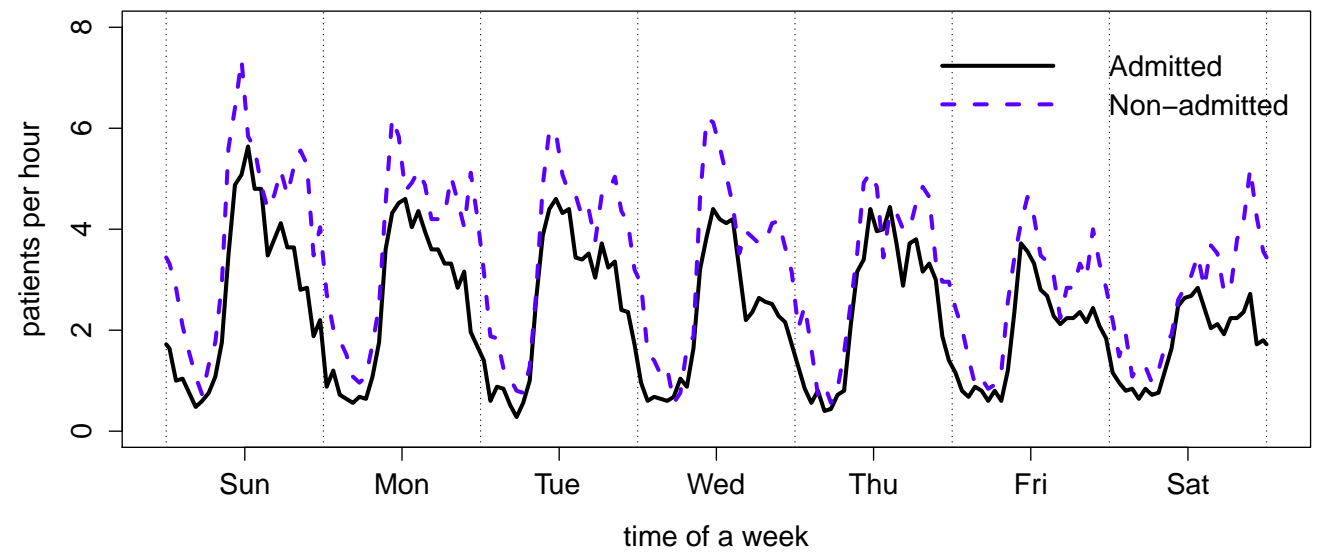

Figure 6: Estimated arrival rates for the admitted and non-admitted patients. (Use datasets 5 and 6.$)$

We also looked at the proportion of patients admitted to the internal ward as a function of their arrival time, denoted by $p(t)$. Figure 7 shows estimates of the proportion of admitted patients by time of day over a single day, 
using all 175 days. Figure 7 presents strong evidence that the probability of admission is indeed time-varying. From a modelling perspective, it is significant that time-dependent, but stochastically independent, thinning also preserves the NHPP property; i.e., if $A$ is an NHPP, then the two separate arrival processes will be NHPP's as well; see Proposition 2.3.2 of [31].

Figure 7: Estimated proportion of admitted patients as a function of the arrival time within each DoW plus the overall average shown by the black solid line (left) and compared to the fitted quadratic function (right). (Use datasets 5 and 6. .)
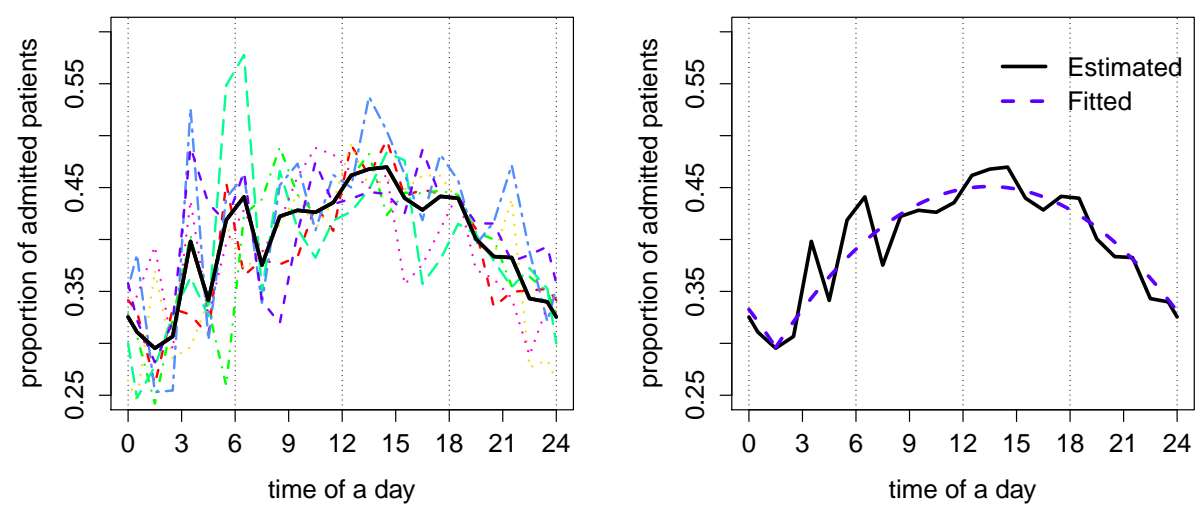

Furthermore, we use least squares to fit a quadratic function to $p(t)$ with a maximum at 2:30 pm. Figure 7 (right) shows fitted function, which is $\hat{p}(t)=-0.001082(x-13.5)^{2}+0.451996$, where $x=((t-1.5) \bmod 24)+1.5$ and $t \in[0,24]$. The modulus function is used to treat the data as periodic with a daily cycle.

\subsection{Summary: Full Model of the ED Arrival Process}

We combine the analysis in the previous subsections to develop a full arrival process model that can be used in simulation studies. First, the daily totals for the number of arrivals are modelled as independent random variables with a Gaussian distribution, as determined by the single factor Gaussian model in (2). Then, given the daily totals, the arrival process is modelled as an NHPP, which means that the given random daily number of arrivals are treated as i.i.d. random variables over the entire day with a pdf proportional to the estimated arrival rate function for that day. We refer to that arrival process model as $M_{t}^{T}$. Finally, a patient that arrives at time $t$ is 
admitted with probability $p(t)$, estimated by the quadratic function above. We conduct simulation experiments using the model in $\S 6$.

However, because we found only moderate overdispersion of the arrival process within each day and only limited dependence among the successive daily totals, our statistical analysis can be interpreted as providing support for an ordinary NHPP $\left(M_{t}\right)$ arrival process model. However, we did observe significant deviations from an NHPP, as is evident from the means and variances in Table 2. More importantly, we think that the two-time-scale arrival-process model introduced here is a useful framework to study potential deviations from an NHPP model. To directly fit an NHPP is to ignore the model fit question entirely.

We also point out that the two-time-scale model is easy to use in simulation models. The model has the advantage over the NHPP that it allows simulation studies of the impact of overdispersion of the daily-total distribution and dependence among the successive daily totals on the ED performance, because these features can be directly included in the model.

\section{Length of Stay}

In this section, we investigate the patient LoS distribution. (In doing so, recall that the LoS is declared over when the admission decision is made, i.e., whether or not to admit the patient to an internal ward; an admitted patient may still be in the ED waiting to be transferred to an internal ward.) We first find that the LoS distribution should be regarded as time-varying. Then we introduce a discrete-time analysis to expose the structure in more detail. We discuss an alternative state-dependent LoS distribution in $\S 4.5$.

\subsection{Failure of the $G_{t} / G I / \infty$ Aggregate Model}

It is common to directly examine the LoS distribution, as if that should be a natural primitive. For modelling, that means that the LoS of successive patients would be modelled as i.i.d. random variables with that distribution. Given that perspective, we started by estimating the overall LoS distribution; we refer to the appendix for the details. That was accomplished by looking at the difference between the exit time and entrance time of each patient. A more elaborate model of what happens in between arrival and departure was not possible, because such extra information was not included in the data.

It also turns out to be highly significant that the departure or exit time was defined as the time that the ED doctor made the decision whether or not 
to admit the patient. Thus, for admitted patients, the additional time until the transfer to the Internal Ward (IW) was not included in the data. Thus, we were unable to directly study the important problem of ED boarding (the extra delay between the admission decision and the patient being transferred to the IW).

Given that perspective, a natural aggregate model for the ED would be an $M_{t} / G I / \infty$ or $G_{t} / G I / \infty$ infinite-server queue, combining an arrival process with a time-varying arrival-rate function with the patient LoS modelled by a sequence of independent and identically distributed (i.i.d.) service times with a general cumulative distribution function (cdf) $G$.

To see if these models with GI LoS times are approximately appropriate, we calculated the time-varying departure rate $\delta(t)$ and the mean occupancy level $m(t) \equiv E[Q(t)]$ in the $G_{t} / G I / \infty$ model using Theorem 1 of [22] together with the estimated arrival-rate function $\lambda(t)$ and LoS cdf $G$. (As emphasized by $\S 5$ of [32], these formulas apply to $G_{t}$ as well as $M_{t}$ arrivals, and so also apply to $M_{t}^{T}$ arrivals, as assumed in §3.6.) Figure 8 compares the directly estimated departure rate and mean occupancy to the indirect estimator exploiting the model. Figure 8 shows that the model with GI LoS does not nearly approximate the actual departure rate and mean occupancy levels. Especially striking is the surge in departures at the end of the day, around midnight, which is totally missed by the $M_{t} / G I / \infty$ model. In closing, we remark that Figure 8 parallels Figure 3 in [7]. There it is emphasized that the peak occupancy lags after the peak arrival rate, which can be seen from Figure 8 as well.

\subsection{The Time-Varying LoS Distribution}

To directly see the time-varying structure of the LoS distribution, we looked at a box-plot of the LoS for each hour; see [33] for backgound. The time-varying behavior of the LoS can be seen from a week view (see the appendix), but is especially clear in a day view, as shown in Figure 9. The boxes show the $25 \%$ and $75 \%$ percentiles, while the blue diamonds are the means and the black bars are the medians. Consistent with intuition, the LoS is longer for patients arriving after midnight, when there are fewer staff. The LoS also tends to be somewhat less for arrivals in the evening. This may be explained by extra effort to release non-admitted patients by midnight, which we will discuss soon.

Given the time-dependence in the LoS distribution, we decided to do a careful analysis in discrete time. For that purpose, let $X_{k, j}$ be the number of 

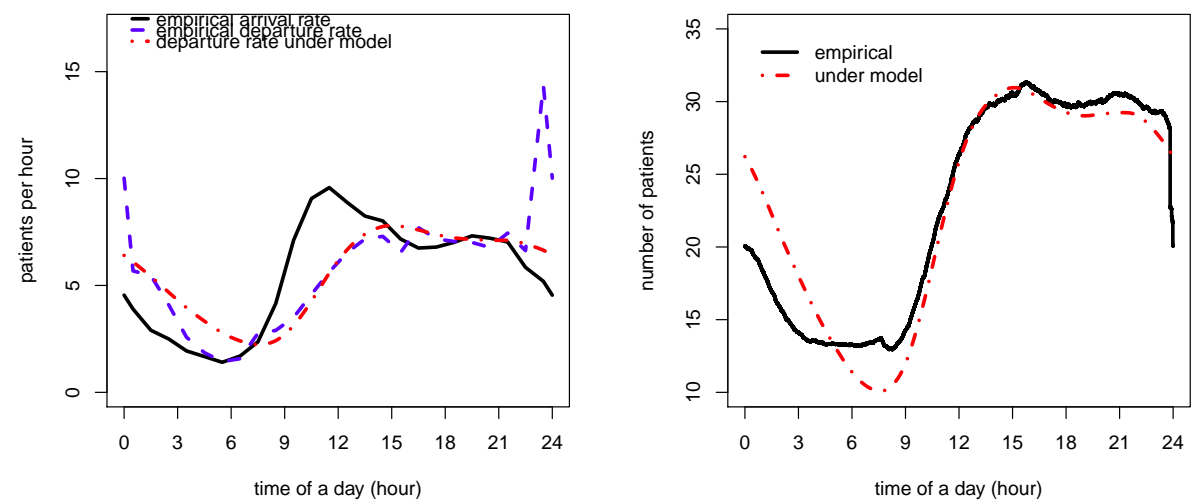

Figure 8: A comparison of direct estimates of the time-varying departure rate $\delta(t)$ (left) and the mean occupancy level $m(t)$ (right) at the ED to indirect estimates based on the $M_{t} / G I / \infty$ model using the estimated arrival rate and LoS ecdf (Use dataset 3.)

arrivals in discrete time period $k$ that have a LoS of $j$ time periods, i.e., that depart in discrete time period $k+j, j \geq 0$. We let a discrete time period be one hour. We still focus on the time period from Dec. 5, 2004, to May 28, 2005 (25 weeks, 175 days), so we make 00:00-01:00 on Dec. 52004 to be discrete time period $k=1$. In order to have the correct number of patients in the system, we only count patients who entered or left the system in that time period and have a reasonable LoS; i.e. we use dataset 4 through this part. We have to include 1 extra day (Dec. 4, 2004) at the beginning to let $X$ include all the patients we focused on. The longest LoS is less than 37 hours after cleaning the data. So our $X$ matrix has dimension $(24 * 176) \times 37$, i.e. for $X_{k, j},-23 \leq k \leq 24 * 175,0 \leq j \leq 36$. The full $X$ matrix is displayed in the appendix.

Let $A_{k}$ and $D_{k}$ be the number of arrivals and departures in the time period $k$. Then we have

$$
A_{k}=\sum_{j=0}^{36} X_{k, j} \quad \text { and } \quad D_{k}=\sum_{j=0}^{36} X_{k-j, j}
$$

where we assume $X_{k, j}=0$ for all $k, j$ except $-23 \leq k \leq 24 * 175,0 \leq j \leq 36$.

Now we assume a periodic structure over successive periods of $d$ discrete times. We assume that we have sufficient data to estimate averages over $n$ 


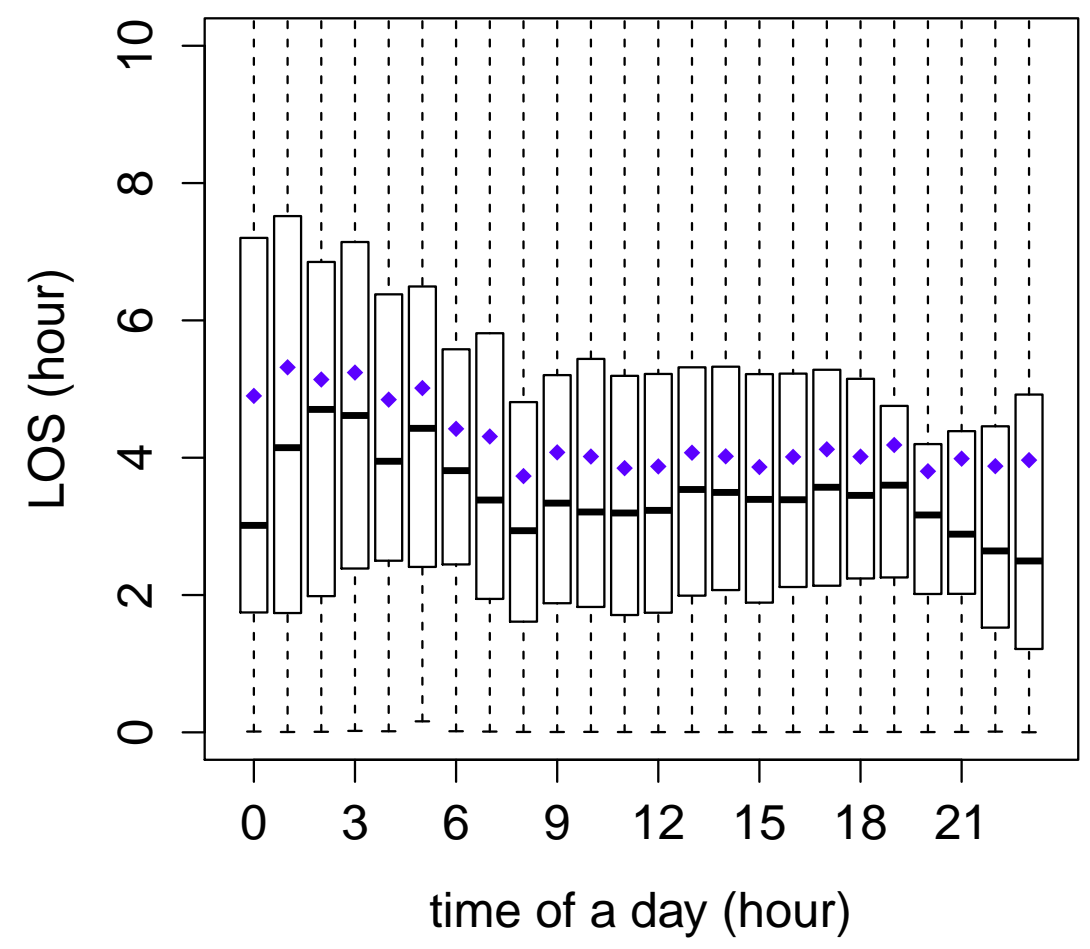

Figure 9: A box plot of the LoS distribution by hour of the day. The blue diamonds are the means, while the black bars are the medians (Use dataset 3.)

periods, containing $n d$ discrete time periods. Specifically, if we consider a period to be 1 week, then we have $n=25$ and $d=7 * 24$; if we consider a period to be 1 day, then we have $n=175=7 * 25$ and $d=24$.

In this periodic setting, we construct averages. In particular, let

$$
\bar{A}_{k}=n^{-1} \sum_{m=1}^{n} A_{(m-1) d+k}, \quad \bar{D}_{k}=n^{-1} \sum_{m=1}^{n} D_{(m-1) d+k}
$$

and

$$
\bar{X}_{k, j}=n^{-1} \sum_{m=1}^{n} X_{(m-1) d+k, j},
$$


for $1 \leq k \leq d$ and $0 \leq j \leq 36$. The $\bar{X}$ matrix for $d=24$ is shown in the appendix. Table 5 shows part of the transpose of the $\bar{X}$ matrix; i.e., the entry in row $j$ and column $k$ is the average number of arrivals in hour $k$ who had a LoS equal to $j$ hours, so that the bold values correspond to the surge

\begin{tabular}{|c|c|c|c|c|c|c|c|c|c|}
\hline & 7 & 8 & 9 & 10 & 11 & 12 & 13 & 14 & 15 \\
\hline 1 & 0.131 & 0.246 & 0.491 & 0.817 & 1.069 & 1.263 & 1.194 & 1.006 & 0.680 \\
\hline 2 & 0.234 & 0.366 & 0.709 & 1.194 & 1.560 & 1.543 & 1.446 & 1.211 & 1.114 \\
\hline 3 & 0.314 & 0.417 & 0.754 & 1.177 & 1.691 & 1.554 & 1.286 & 1.343 & 1.549 \\
\hline 4 & 0.263 & 0.337 & 0.623 & 1.040 & 1.114 & 1.154 & 1.331 & 1.257 & 1.171 \\
\hline 5 & 0.263 & 0.171 & 0.320 & 0.669 & 0.703 & 1.257 & 0.840 & 1.011 & 0.874 \\
\hline 6 & 0.189 & 0.194 & 0.217 & 0.411 & 0.697 & 0.657 & 0.583 & 0.594 & 0.651 \\
\hline 7 & 0.091 & 0.120 & 0.154 & 0.400 & 0.394 & 0.366 & 0.474 & 0.423 & 0.343 \\
\hline 8 & 0.023 & 0.051 & 0.171 & 0.246 & 0.274 & 0.257 & 0.263 & 0.211 & 0.211 \\
\hline 9 & 0.029 & 0.103 & 0.074 & 0.131 & 0.211 & 0.206 & 0.211 & 0.137 & $\mathbf{0 . 4 4 6}$ \\
\hline 10 & 0.006 & 0.051 & 0.017 & 0.109 & 0.149 & 0.097 & 0.069 & $\mathbf{0 . 3 8 3}$ & 0.051 \\
\hline 11 & 0.023 & 0.017 & 0.023 & 0.080 & 0.051 & 0.086 & $\mathbf{0 . 2 6 9}$ & 0.034 & 0.051 \\
\hline 12 & 0.017 & 0.034 & 0.029 & 0.029 & 0.046 & $\mathbf{0 . 2 4 6}$ & 0.051 & 0.011 & 0.011 \\
\hline 13 & 0.011 & 0.017 & 0.006 & 0.023 & $\mathbf{0 . 3 6 6}$ & 0.006 & 0.029 & 0.011 & 0.006 \\
\hline 14 & 0.000 & 0.006 & 0.000 & $\mathbf{0 . 2 3 4}$ & 0.011 & 0.023 & 0.011 & 0.000 & 0.000 \\
\hline 15 & 0.006 & 0.006 & $\mathbf{0 . 1 2 6}$ & 0.006 & 0.000 & 0.017 & 0.000 & 0.000 & 0.011 \\
\hline 16 & 0.000 & $\mathbf{0 . 0 5 7}$ & 0.000 & 0.006 & 0.006 & 0.006 & 0.011 & 0.017 & 0.011 \\
\hline 17 & $\mathbf{0 . 0 3 4}$ & 0.000 & 0.000 & 0.006 & 0.000 & 0.006 & 0.006 & 0.017 & 0.011 \\
\hline 18 & 0.000 & 0.006 & 0.000 & 0.000 & 0.000 & 0.000 & 0.000 & 0.017 & 0.011 \\
\hline
\end{tabular}

Table 5: Part of the transpose of the $\bar{X}$ matrix; i.e., the entry in row $j$ and column $k$ is the average number of arrivals in hour $k$ who had a LoS equal to $j$ hours, so that the bold values correspond to the surge just before midnight (Use dataset 4).

just before midnight

To make the structure more evident, We show some of the cells shadowed and bold. Those diagonally arranged cells correspond to the average number of patients that arrived in the column hour whose row value of LoS made them depart from the ED in the hour before midnight. Table 5 shows that many patients depart from the ED just before midnight. For example, consider the arrival in hour (column) 10. The average number increases from $j=1$ to $j=2$, but then decreases to the low value 0.023 at $j=13$ before jumping up to 0.234 at $j=14$, a value 10 times higher, before declining rapidly toward 0 .

Again, we emphasize that the data we used only provides the entry time and exit time for each patient, where the exit time is when the ED doctor made the admission decision. Evidently there is a change in medical staff at midnight that increases the number of admission decisions just before midnight. 


\subsection{The LoS of the Two Groups}

Just as for the arrival process, we want to study differences in the LoS distribution for the admitted and non-admitted patients. Figure 10 shows the empirical LoS distribution for the two groups without time structure. The admitted patients have a smaller mean LoS but a longer median, because about $7 \%$ of the admitted patients have an extremely low LoS. Evidently, these patients were transfered immediately to the IW's. If we omit the admitted patients whose LoS is less than 2 minutes (657 patients), then the mean LoS of the admitted group increases to 4.30 hours, which is larger than the non-admitted group.

Then we look at the time-varying feature of the LoS for the two groups, again using box plots. Figure 11 shows that the time-varying LoS distribution is more regular for the non-admitted patients. We see quite striking differences for admitted patients before and after midnight.

\subsection{The LoS Model and Occupancy}

Our analysis of the LoS data, leads us to model the LoS distribution as (i) time-dependent and (ii) depending on whether the patient is admitted or not. If we use the $M_{t}^{T}$ two-time-scale arrival process model in $\S 3.6$ and ignore the distinction between the admitted and non-admitted patients, this produces an $M_{t}^{T} / G I_{t} / \infty$ infinite-server aggregate model. Extending it to the two types of patients, the model becomes two independent $M_{t}^{T} / G I_{t} / \infty$ models, again using the arrival process model from $\S 3.6$, one for the admitted patients and another for the non-admitted patients. We would use the separate timevarying LoS distribution for each group. We remark that this independence assumption is an approximation because in fact the two groups are necessarily dependent because they use the same resources.

Even though an infinite-server model was not suggested in [7], the infiniteserver model is consistent with several observations in it. First, in $\S 3.1$ [7] the authors emphasize that the bed capacity of the ED is highly flexible, so that there is effectively unbounded. Second, in Figures 4 and 5 in $§ 3.2 .2$ of [7] the authors observe that a time-varying Gaussian distribution fits the occpancy data well, but that is consistent with the theoretical time-varying Poisson distribution in the time-varying $M_{t} / G I / \infty$ model and the heavytraffic Gaussian approximations for infinite-server models in [34]. 

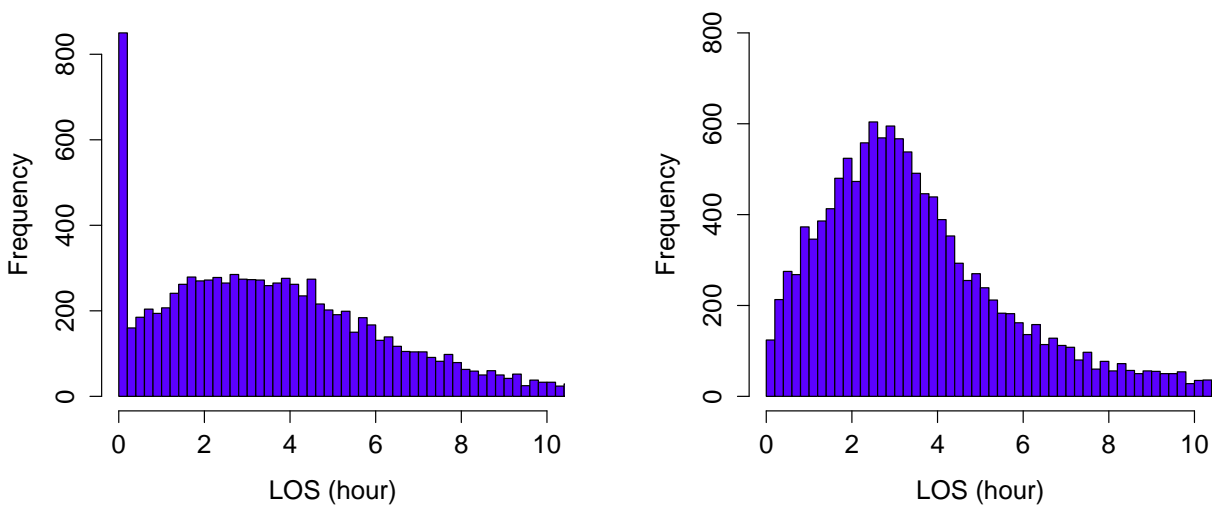

(a) LoS of admitted patients. (x-axis lim- (b) LoS of non-admitted patients. (x-axis ited to 10 hours) limited to 10 hours)

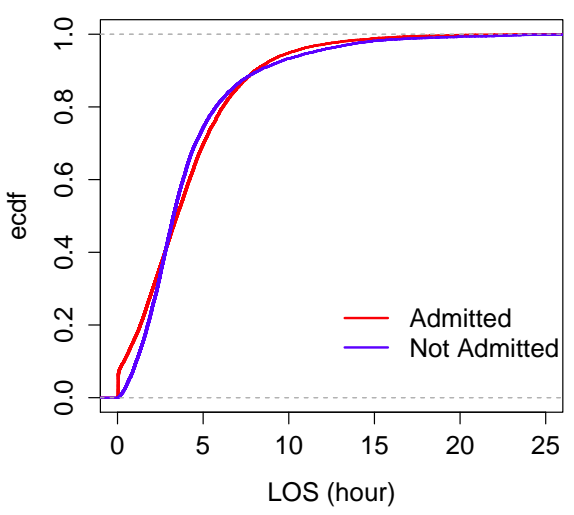

(c) empirical cdf's for the 2 groups.

Figure 10: Estimated LoS distributions of the admitted and non-admitted patients truncated to $[0,10]$. (Use datasets 5 and 6 .)

\subsection{Time Dependence Versus State Dependence}

We have proposed a time-dependent LoS distribution in contrast to the state-dependent LoS distribution proposed in $[7,17]$ and in references cited there. Because there is strong time-dependence in the occupancy level, these two forms of dependence are intimately linked and not easy to separate.

To substantiate that claim, we provide a state-dependent analog of Figure 

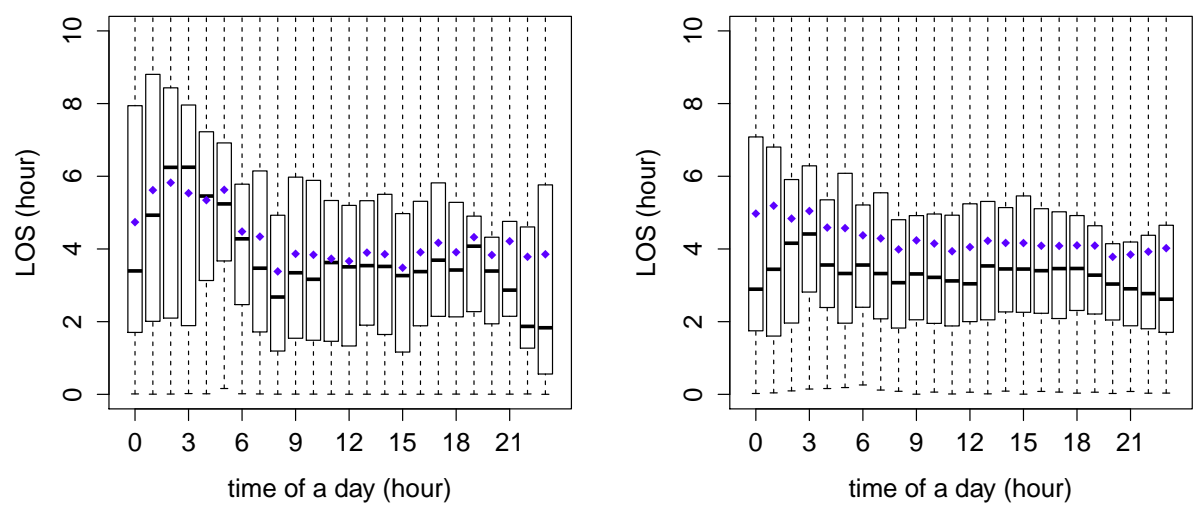

Figure 11: Box plots of the LoS distribution as a function of the arrival time for admitted (left) and non-admitted (right) patients. (Use datasets 5 and 6.)

9 in Figure 12. Figure 12 provides a box plot of the LoS distribution by state, i.e. the number of patients in the ED. We also plot the sample size as a function of the state, shown on the right axis, which shows that there are fewer arrivals when the state is either low or high. From either the mean values (purple diamonds) or the medians (black bars), we see the that the LoS is an increasing function of the ED occupancy.

In general, which model is preferred may depend on the ease of analysis. The state-dependent LoS model might be considered to be more tractable, because it produces a stationary model. Nevertheless, state-dependent models, especially non-Markovian state-dependent models, are not easy to analyze. In fact, a good case can be made that models with time dependence are actually easier to analyze; there is now a substantial literature, e.g., $[14,15,32,35]$.

Both views provide important insight. State dependence shows that ED congestion increases the patient LoS, while time dependence make it easier to connect the results to ED operations and hospital routines, which tend to be driven by the clock much more than the load. In particular, hospital routines usually dictate that hospital admission and release decisions tend to be made at prescribed times.

In particular, for the ED data, we have presented strong evidence for time-dependence as opposed to state-dependence, because of the surge of departures just before midnight on each day, as can be from Figures 8 (left) 


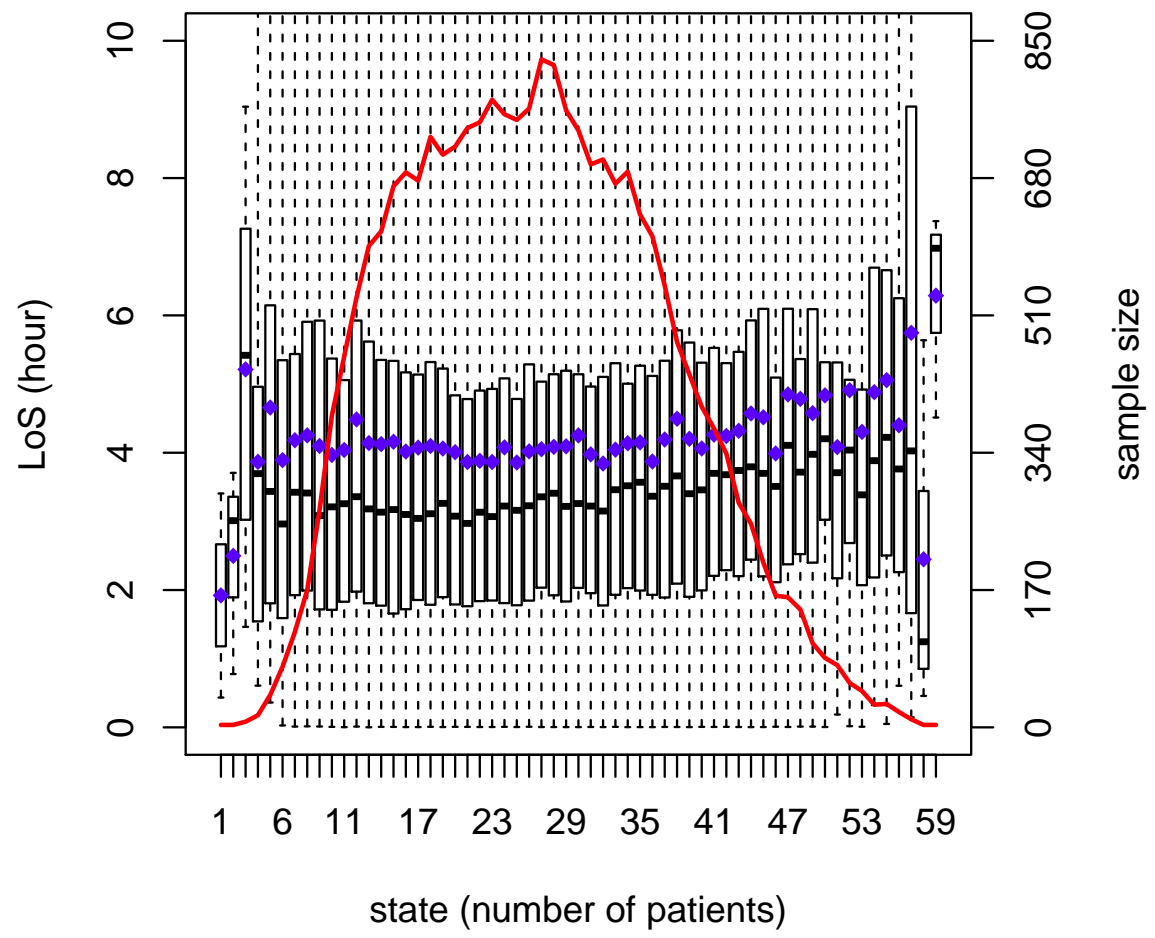

Figure 12: A box plot of the LoS distribution by state, i.e. the number of patients in the ED. The purple diamonds are the means, while the black bars are the medians (Use dataset 3.)

and 17. Table 5 shows that these midnight departures occur for patients that arrive across a wide range of times. Nevertheless, it can be captured by a time-varying LoS distribution.

It remains to carefully examine state-dependent models. Evidently, a state-dependent LoS cannot capture the midnight departure surge, but there are a variety of state-dependent models that might be considered. Presumably, proper state-dependence should take account of the occupancy throughout a patient's LoS, not just at arrival, but that is not easy to implement. 


\section{The Departure process}

In this section, we investigate the departure process from the ED. As a theoretical reference point, for an the $M_{t} / G I_{t} / \infty$ model, the departure process is also an NHPP. We find it useful to look at the departure process and the entire ED in reverse time, so that we can think of the departure process as an arrival process and use the same methods we have used in previous sections. That reverse-time perspective is especially revealing to look at the time-varying proportion of admitted patients and the time-varying LoS, where the time refers to the departure time instead of the arrival time.

\subsection{Daily Totals}

Paralleling $§ 3.1$, we first look at the daily totals of departures, but we provide only a brief overview; see the appendix for the tables and figures.

The reverse-time perspective forces us to change the data a little. Now we consider the patients that left the from Dec. 5, 2004, to May 28, 2005, which is 23,407 patients in total (see Table 1). The mean values for each week and each DoW are almost the same as for the arrivals, but there is a significant difference in the variances. The variance of the total numbers of departures by DoW is higher than for the arrivals. Evidently, there is less regularity in departures than in arrivals.

Again, we fit the Gaussian regression models in (1) and (2) in $§ 3.1$ for the departures. The parameters have the same meaning as before. Table 6 shows the ANOVA results. As before, both the Week factor and the DoW

\begin{tabular}{|c|c|c|c|c|c|}
\hline Factor & Sum of square & df & Mean sum of square & F statistics & P-values \\
\hline Week & 10661 & 24 & 444.2 & 2.19 & $<0.01$ \\
\hline DoW & 56146 & 6 & 9357.6 & 46.22 & $<0.01$ \\
\hline Residuals & 29156 & 144 & 202.5 & & \\
\hline
\end{tabular}

Table 6: ANOVA table for the two-factor model (1) for the departures. (Use dataset 7.)

factor are statistically significant, but the DoW factor explains most of the variance. For the two-factor model, the mean sum of square for the residuals is $\hat{\sigma}^{2}=202.5=14.23^{2}$, which is higher than that of the arrival process. The variance-to-mean ratio is $202.5 / 133.8=1.51$. If we omit the Week factor and consider the single factor model. Then the mean sum of square for the residuals is $(10661+29156) /(24+144)=237.0$ and the variance-to-mean ratio is $237.0 / 133.8=1.77$. 


\subsection{Departure Pattern Within Each Day}

Now we turn to the time structure of departure rate within days. Figure 13 shows the reverse-time view. Paralleling and amplifying Figure 8, Figure 13 shows clearly that the departure rate has midnight surges and that the peaks are increasing over the week.

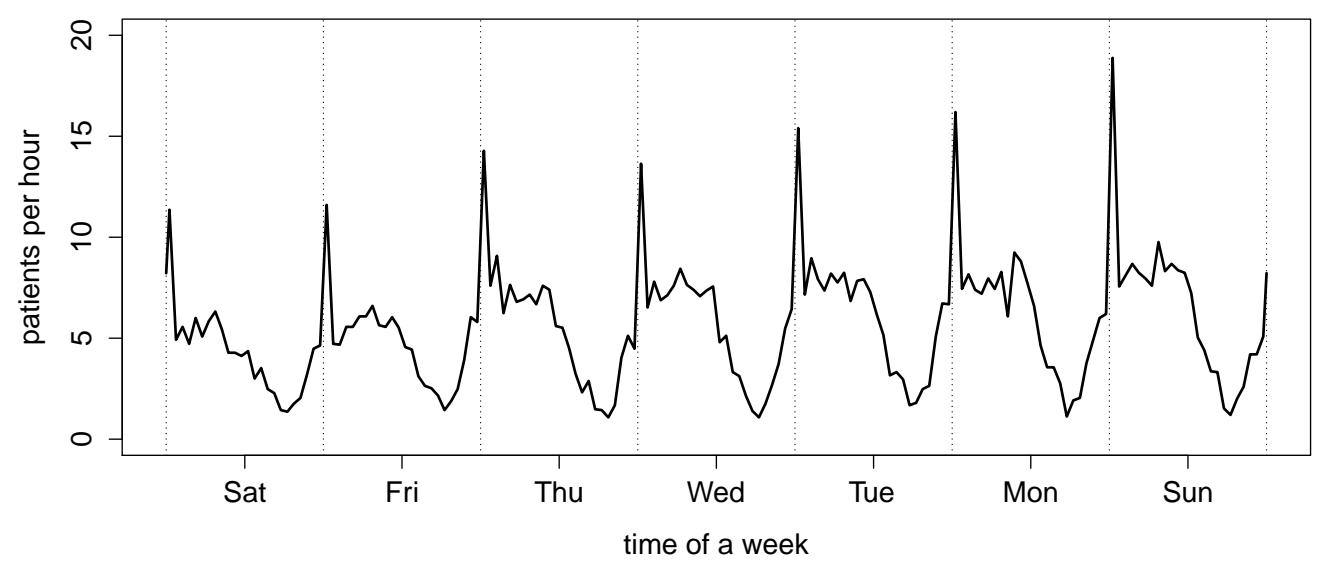

Figure 13: Estimated departure rate at the ED in reverse time. (Use dataset 7.)

As before, we divided the patients into two groups according to the admission decision. (See Table 1 for basic statistics.) Figure 14 shows the time-varying proportion of admitted patients as a function the departure time. We see that the proportion of admitted patients is extremely low at 78 a.m. of each day. Evidently, admission decisions at that time are postponed until new doctors arrive after morning staff changes.

Figure 15 presents box plots of the LoS distribution as a function of the departure time (in reverse-time perspective) for admitted (left) and nonadmitted (right) patients. We see that the midnight surge is caused by the non-admitted patients, and that the LoS of non-admitted patients are more influenced by time of the day.

\section{Comparison with Simulation}

In this section we conduct simulations to substantiate our model. 


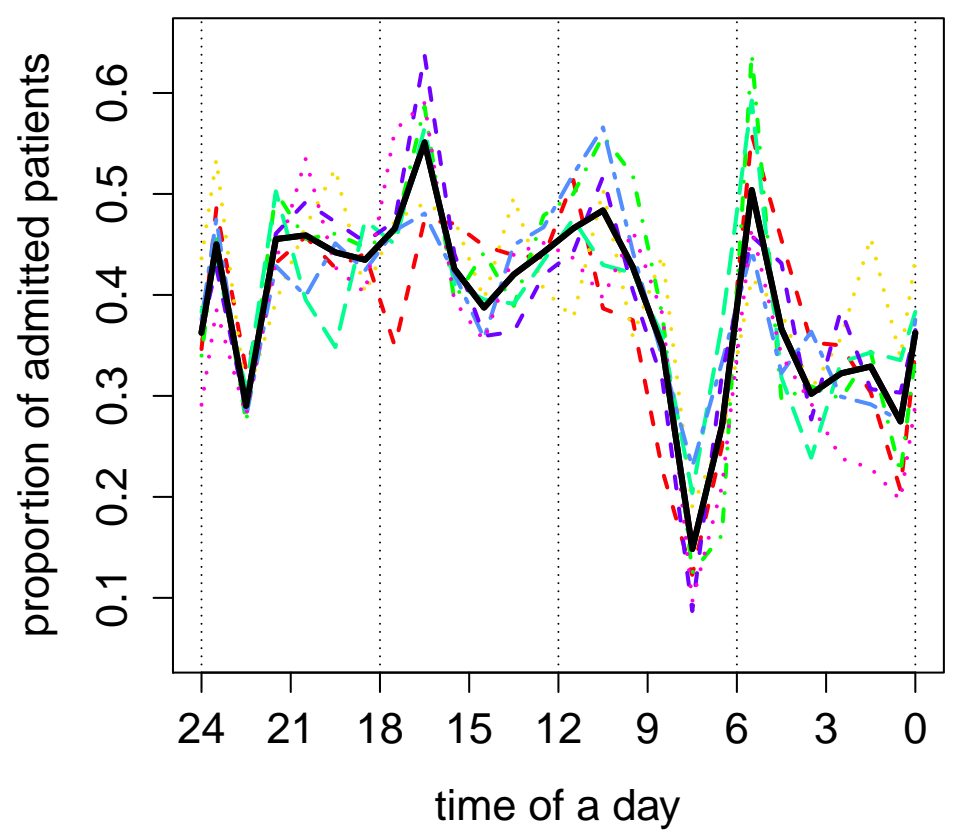

Figure 14: Estimated time-varying proportion of admitted patients as a function of the departure time from the ED over a day, for each DoW and overall, combining all days together (black solid line). (Use datasets 8 and 9.)
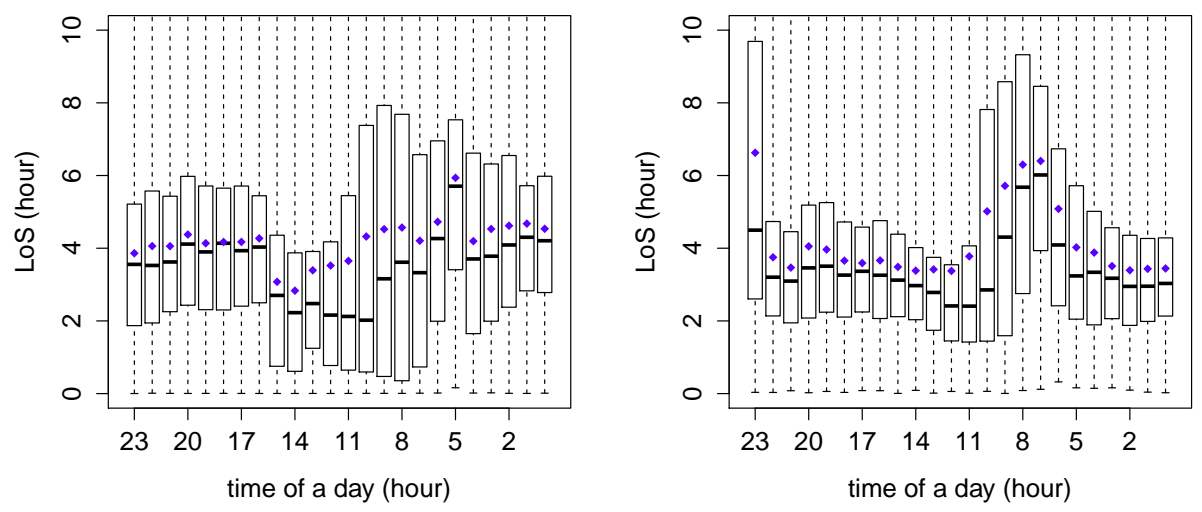

Figure 15: Box plots of the LoS distribution as a function of the departure time (in reverse time) for admitted (left) and non-admitted (right) patients. (Use datasets 5 and 6.) 


\subsection{Comparing Alternative LoS Models}

We conduct simulation experiments with our model to see how it represents the data. First, we focus on the LoS model. To do so, we use the original arrival data. We repeat the 25 weeks 40 times, so that the sample size is 1000 weeks. To examine alternative LoS models, we treat them in three different ways: (A) The first option is GI, i.e., we assume that the LoS distribution is not time-varying; we use the overall estimated cdf; (B) The second option is $G I_{t}$ but with a day view; i.e., we assume that the LoS distribution is time-varying over each day; we use the estimated time-varying cdf depending on the arrival time within a day; (C) The third option is also $G I_{t}$ but with a week view; i.e., we assume that the LoS distribution is timevarying over each week; we use the estimated time-varying cdf depending on the arrival time within a week.

Figures 16 and 17 compare the indirect model estimates to direct simulation estimates of the time-varying expected occupancy $m(t)$ and the timevarying departure rate $\delta(t)$, respectively, based on each of these three LoS models.

The top plots of Figures 16 and 17 show the consequence of ignoring the time-varying LoS distribution. Consistent with Figure 8, Figure 16 shows that the GI LoS model significantly underestimates the occupancy at the end of the day, before midnight, and overestimates it at the beginning of the day, after midnight, while Figure 17 shows that the GI LoS model completely misses the midnight surge of departures.

The middle plots (B) of Figures 16 and 17 show that the $G I_{t}$ LoS model with a day view does much better than the GI model, capturing the midnight surge in departures. Nevertheless, there is a clear gap between the mean occupancy curves. Remarkably, the bottom plots (C) of Figures 16 and 17 show that the $G I_{t}$ LoS model with a week view show near-perfect agreement.

\subsection{Evaluating the Full Model}

We obtain a full ED model when we (i) incorporate the $M_{t}^{T}$ arrival model summarized in $\S 3.6$, (ii) divide the arrivals into the two groups, admitted and non-admitted, using independent thinning according to the time-varying probability $p(t)$ estimated in $\S 3.5$, and (iii) when we use a separate LoS model for each group.

We repeated the three experiments Figures 16 and 17 using the full model. We applied the three LoS models to each group separately. The new simu- 

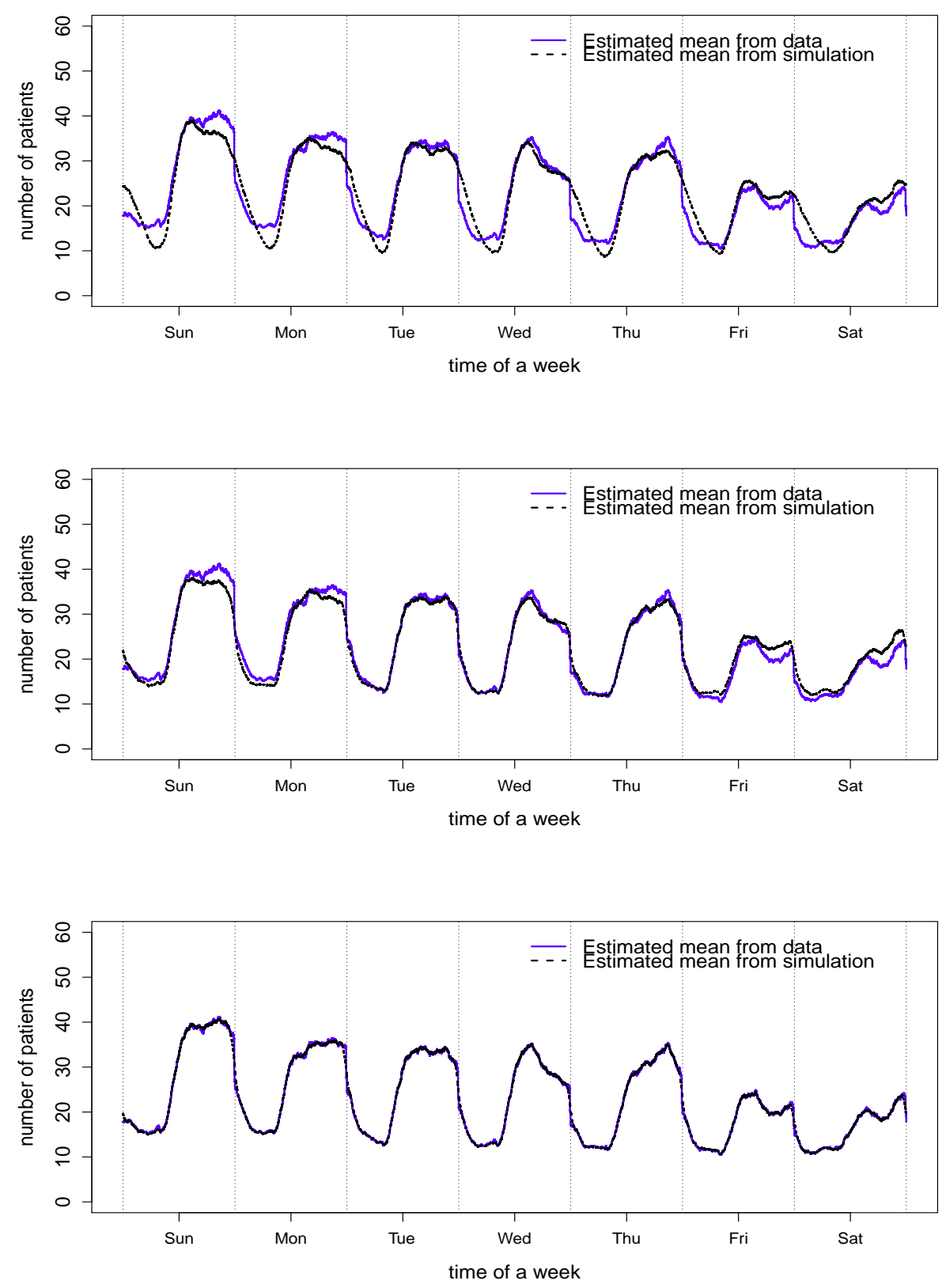

Figure 16: Simulation estimates of the time-varying expected occupancy $m(t)$, based on the arrival data plus three LoS models: (A) $G I$ (top), (B) $G I_{t}$ with day view (middle) and (C) $G I_{t}$ with week view (bottom) 

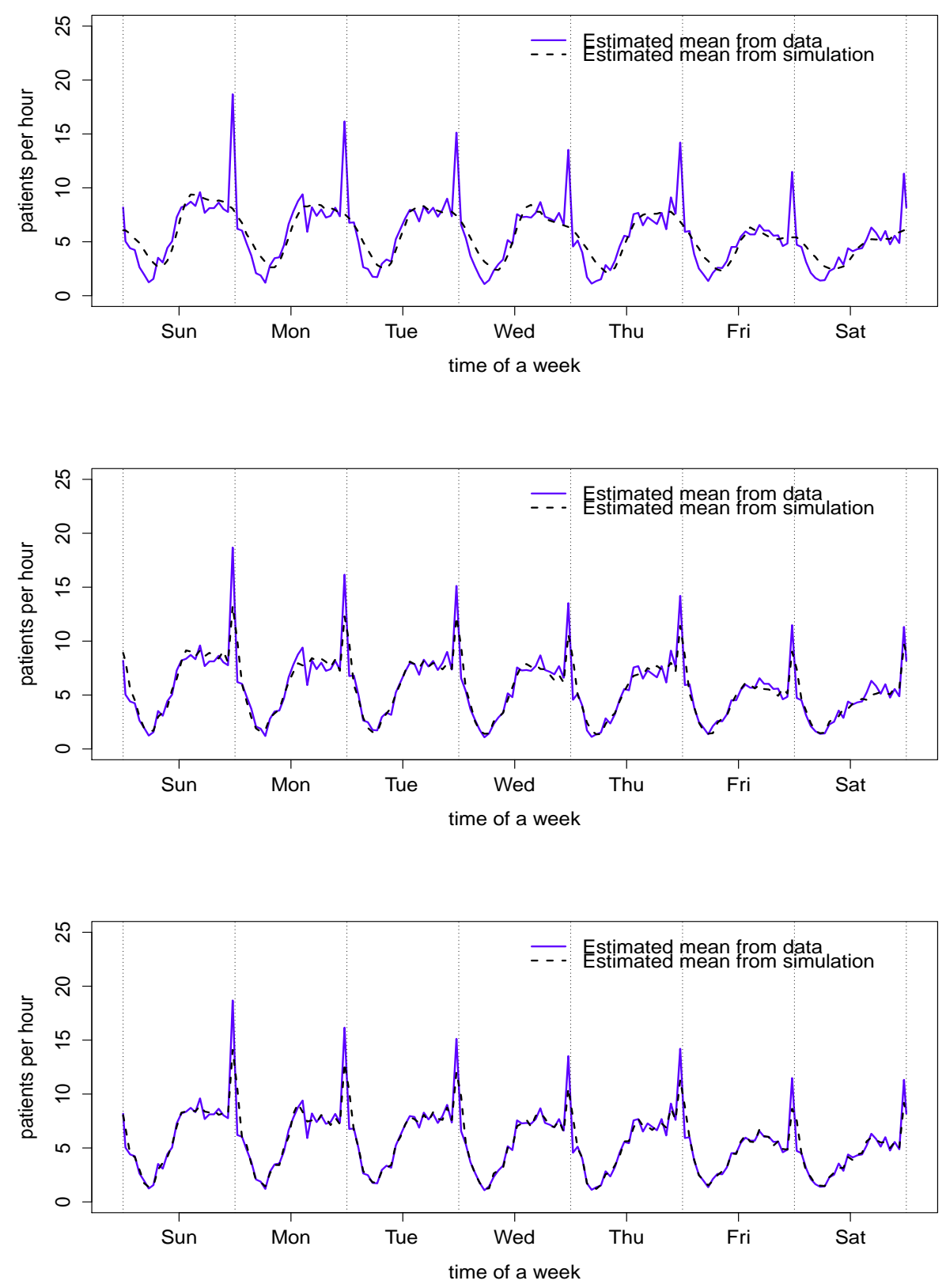

Figure 17: Simulation estimates of the time-varying departure rate $\delta(t)$ based on the arrival data plus three LoS models: (A) $G I$ (top), (B) $G I_{t}$ with day view (middle) and (C) $G I_{t}$ with week view (bottom) 
lation results look virtually identical to Figures 16 and 17, and so they are only shown in the appendix.

\subsection{The Time-Varying Little's Law}

The spectacular agreement between the simulation estimates for case $(\mathrm{C})$ were initially puzzling. However, we find that this can be explained in large part by the time-varying Little's law (TVLL), as in [23, 24]. The TVLL Little's law applies to a $G_{t} / G_{t} / \infty$ model and thus to our $M_{t}^{T} / G I_{t} / \infty$ model. The discrete-time study in this paper motivated us to also consider a discretetime version of the TVLL. We intend to discuss the discrete-time TVLL and the implications of the TVLL in [36]. Briefly, the implications are that we should regard the accurate prediction of the average occupancy given the $G_{t} / G I / \infty$ aggregate model as a data consistency check rather than a genuine prediction, when we estimate the average occupancy from the same data used to fit the model.

\section{Conclusions}

We studied a 25-week portion of the ED data used in the patient flow study by Armony et al. [7]. We carefully studied the arrival process to the ED and the patient LoS distribution, reaching several important conclusions.

First, for the arrival process, we think that it is helpful to use the twotime-scale approach, in which we first look at daily totals and then look at the arrival process within each day conditional on the daily totals, which leads to the arrival process model summarized in $\S 3.6$. In $\S 3.1$ we examined factor regression models for the daily totals, and adopted the single-factor model in (2), which expresses the daily totals as an expected value depending on the day of the week (DoW) plus a mean-0 Gaussian distribution with a variance that is determined by the regression. This directly leads to a model of independent daily totals with a Gaussian distribution depending on the DoW. The two-time-scale model is useful, because it provides a useful framework for future research. It is natural to next look for improvements to the model of daily totals by exploiting (i) time-series models, (ii) forecasting methods and (iii) more context knowledge to capture the dependence in successive daily totals. Preliminary investigation revealed positive dependence among the residuals, as indicated in $\S 3.2$ and expanded upon in the appendix [25]. With further work, it may be possible to capture the dependence over multiple days shown in Figure 5 (first two plots). 
We studied the time-varying arrival rate in $\S 3.3$. We concluded that it is important to take a week view, as shown in Figure 3, rather than the common day view. An important new finding is the dependence of decision to admit a patient from the ED into an internal ward upon the time of arrival, discussed in §3.5. (It still remains to find a good explanation.) Even though the admission decision cannot be known in advance for individual patients, we can exploit the time-dependence in the observed admission decisions to model these two groups of patients differently. Finally, we examined the stochastic variability in the arrival process in $\S 3.4$ and found support for the two-time-scale model, where conditional on the daily totals, the arrival within the day can be modelled as an NHPP. We denote this arrival process as $M_{t}^{T}$.

Second, we analyzed the patient length-of-stay (LoS) distribution in $§ 4$. We concluded that this too should depend on the arrival time. We discuss alternative state-dependent models as in [7, 17] in $§ 4.5$. Figures 8, 16 and 17 dramatically show the consequence of ignoring this time-varying feature. Of course, it is desirable to do a more detailed modelling of the flow within the ED, presumably with a queueing network model, so that the overall LoS distribution can be analyzed through its component parts, but the available data did not permit that. Even after that is done, an aggregate model should be helpful for comparison.

Combining the arrival process model in $\S 3$ and the LoS model in $\S 4$, we obtain the proposed $M_{t}^{T} / G I_{t} / \infty$ time-varying infinite-server aggregate model of the ED. This model becomes expanded to two independent such infinite-server models if we separately model the admitted and non-admitted patients, with independence following from the independent thinning of an NHPP. This model can be used for capacity planning and for comparison in more detailed queueing network models of the ED.

We think it is also important to analyze the departure process from the $\mathrm{ED}$, which we do in reverse time in $\S 5$. The departure rate function in Figure 13 clearly shows the midnight surge, which can be missed from other views. Figures 14 and 15 show that the admission decision and the LoS both depend on the departure time as well as on the arrival time.

Finally, we compared our model to simulation in $\S 6$. We found remarkable agreement in the average occupancy level and the departure rate, but discovered that these high-quality approximations can largely be explained by the time-varying Little's law in [23, 24], as we plan to discuss in [36].

There are many remaining problems for future research. First, it remains 
to carefully examine the surge of departures before midnight each day and its cause. Second, it remains to examine the ability of the model to predict the future. Third, it remains to find and examine more extensive data sets that include: (i) the full LoS until the patient secures a bed in the internal ward, (ii) the operational steps within the ED, and (iii) the use and availability of additional resources, such as doctors and nurses. Finally, it remains

to compare and contrast state-dependent and time-dependent models more generally.

Acknowledgement. We thank Avishai Mandelbaum, Galit Yom-Tov and their colleagues at the Technion IE\&M Laboratory for Service Enterprise Engineering (SEELab) for providing access to the Israeli Rambam hospital data. We thank NSF for research support through CMMI 1265070 and 1634133.

\section{References}

[1] R. B. Fetter, J. D. Thompson, The simulation of hospital systems, Operations Research 13 (5) (1965) 689-711.

[2] M. L. Brandeau, F. Sainfort, W. P. Pierskalla, Operations research and health care: a handbook of methods and applications, Springer Science \& Business Media, 2004.

[3] B. T. Denton, Handbook of Healthcare Operations Management, 2nd Edition, Springer, 2013.

[4] R. W. Hall, Handbook of Healthcare System Scheduling, Springer, 2012.

[5] R. W. Hall, Patient flow: reducing delay in healthcare delivery, 2nd Edition, Springer Science \& Business Media, 2013.

[6] R. S. Kaplan, M. E. Porter, How to solve the cost crisis in healthcare, Harvard Business Review 89 (2011) 46-64.

[7] M. Armony, S. Israelit, A. Mandelbaum, Y. Marmor, Y. Tseytlin, G. Yom-Tov, Patient flow in hospitals: a data-based queueing-science perspective, Stochastic Systems 5 (1) (2015) 146-194.

[8] M. A. Ahmed, T. M. Alkhamis, Simulation optimization for an emergency department healthcare unit in kuwait, European Journal of Operations Research 198 (2009) 936-942. 
[9] A. M. D. Bruin, A. C. van Ossum, M. C. Visser, G. M. Koole, Modeling the emergency cardiac in-patient flow: an application of queueing theory, Health Care Management Science 10 (2007) 125-137.

[10] L. V. Green, J. Soares, J. F. Giglio, R. A. Green, Using queueing theory to increase the effectiveness of emergency department provider staffing, Academic Emergency Medicine 13 (2006) 61-68.

[11] S. Maman, Uncertainty in the demand for service: The case of call centers and emergency departments, Master's thesis, Technion-Israel Institute of Technology (2009).

[12] D. Sinreich, Y. Marmor, Emergency department operations: the basis for developing a simulation tool, IIE Transactions 37 (2005) 233-245.

[13] T. Collings, C. Stoneman, The $M / M / \infty$ queue with time-varying arrival and departure rates, Operations Research 24 (4) (1976) 760-773.

[14] W. Whitt, Offered load analysis for staffing, Manufacturing and Service Operations Management 15 (2) (2013) 166-169.

[15] L. V. Green, P. J. Kolesar, W. Whitt, Coping with time-varying demand when setting staffing requirements for a service system, Production Oper. Management 16 (2007) 13-29.

[16] P. Shi, M. C. Chou, J. G. Dai, D. Ding, J. Sim, Models and insights for hospital inpatient operations: time-dependent ed boarding time, Management Science 62 (1) (2016) 1-28.

[17] R. J. Batt, C. Terwiesch, Doctors under load: an empirical study of state-dependent service times in emergency care, the Wharton School, the University of Pennsylvania, Philadelphia, PA 19104 (2012).

[18] S.-H. Kim, P. Vel, W. Whitt, W. C. Cha, Poisson and non-Poisson properties in appointment-generated arrival processes: the case of an endrocrinology clinic, Operations Research Letters 43 (2015) 247-253.

[19] S. Kim, W. Whitt, Are call center and hospital arrivals well modeled by nonhomogeneous Poisson processes?, Manufacturing and Service Oper. Management 16 (3) (2014) 464-480. 
[20] S. Kim, W. Whitt, Choosing arrival process models for service systems: Tests of a nonhomogeneous Poisson process, Naval Research Logistics 17 (2014) 307-318.

[21] M. Ramakrishnan, D. Sier, P. G. Taylor, A two-time-scale model for hospital patient flow, IMA Journal of Management Mathematics 16 (2005) 197-215.

[22] S. G. Eick, W. A. Massey, W. Whitt, The physics of the $M_{t} / G / \infty$ queue, Oper. Res. 41 (1993) 731-742.

[23] D. Bertsimas, G. Mourtzinou, Transient laws of nonstationary queueing systems and their applications, Queueing Systems 25 (1997) 315-359.

[24] B. H. Fralix, G. Riano, A new look at transient versions of Little's law, Journal of Applied Probability 47 (2010) 459-473.

[25] W. Whitt, X. Zhang, A data-driven model of an emergency department, appendix: Additional details, Columbia University, http://www.columbia.edu/ ww2040/allpapers.html (2016).

[26] M. H. Kutner, C. Nachtsheim, J. Neter, Applied linear regression models, 4th Edition, McGraw-Hill/Irwin, 2004.

[27] D. J. Daley, D. Vere-Jones, An Introduction to the Theory of Point Processes, 2nd Edition, Vol. II, Springer, Oxford, U. K., 2008.

[28] D. R. Cox, P. A. W. Lewis, The Statistical Analysis of Series of Events, Methuen, London, 1966.

[29] K. W. Fendick, W. Whitt, Measurements and approximations to describe the offered traffic and predict the average workload in a singleserver queue, Proceedings of the IEEE 71 (1) (1989) 171-194.

[30] S. Kim, W. Whitt, The power of alternative Kolmogorov-Smirnov tests based on transformations of the data, columbia University, http://www.columbia.edu/ ww2040/allpapers.html (2013).

[31] S. M. Ross, Stochastic Processes, 2nd Edition, Wiley, New York, 1996. 
[32] O. B. Jennings, A. Mandelbaum, W. A. Massey, W. Whitt, Server staffing to meet time-varying demand, Management Sci. 42 (1996) 13831394.

[33] W. Härdle, L. Simar, Applied multivariate statistical analysis, 3rd Edition, Vol. 22007, Springer, 2012.

[34] G. Pang, W. Whitt, Two-parameter heavy-traffic limits for infiniteserver queues, Queueing Systems 65 (2010) 325-364.

[35] J. A. Schwarz, G. Selinka, R. Stolletz, Performance analysis of timedependent queueing systems: survey and classification, Omega 63 (2016) 170-189.

[36] W. Whitt, X. Zhang, The periodic Little's law and its application to an emergency department, in preparation, Columbia University, http://www.columbia.edu/ ww2040/allpapers.html (2016). 